\title{
EXTREMAL FUNCTIONS FOR MOSER-TRUDINGER TYPE INEQUALITY ON COMPACT CLOSED 4-MANIFOLDS
}

\author{
YUXIANG LI, CHEIKH BIRAHIM NDIAYE
}

\begin{abstract}
Given a compact closed four dimensional smooth Riemannian manifold, we prove existence of extremal functions for Moser-Trudinger type inequality. The method used is Blow-up analysis combined with capacity techniques.
\end{abstract}

Keywords: Moser-Trudinger inequality, Blow-up analysis, Capacity, Extremal function, Green function.

2000 Mathematics Subject Classification: 46E35, 26D10

\section{Contents}

1. Introduction 1

2. Notations and Preliminaries 4

3. Proof of Theorem $1.1 \quad 6$

3.1. Concentration behavior and profile of $u_{k} \quad 6$

3.2. Pohozaev type identity and application 10

3.3. Blow-up analysis 13

3.4. Capacity estimates 17

3.5. The test function 25

3.6. Proof of Theorem $1.1 \quad 27$

4. Proof of Theorem $1.2 \quad 27$

References $\quad 28$

\section{INTRODUCTION}

It is well-known that Moser-Trudinger type inequalities are crucial analytic tools in the study of partial differential equations arising from geometry and physics.

In fact, much work has been done on such inequalities and their applications in the last decades, see for example, [1], [3], [4], [6], [8], [18], [22], and the references therein.

There are two important objects in the study of Moser-Trudinger type inequalities: one is to find the best constant and the other is to determine whether there exist extremal functions.

For the best constant there are the celebrated work of Moser[19] and the extension to higher order derivatives by Adams [1] on flat spaces. In the context of curved spaces Fontana has extended the results of Adams, see [9].

To mention results about extremal functions, we cite the results of Carleson and Chang [5], Flucher [10] and Lin [16] in the Euclidean case and the results of Li [14], [15] in the curved one. In [14] and [15] the author have proved the existence of an extremal function for the classic MoserTrudinger inequality on a compact manifold under a constraint involving only the first derivatives.

In this paper, we will extend the results of $\mathrm{Li}$ to a compact closed four dimensional smooth Riemannian manifold under a constraint involving the Laplacian. More precisely we prove the following Theorems

Date: 11 th June 2021. 
Theorem 1.1. Let $(M, g)$ be a compact closed smooth 4-dimensional Riemannian manifold. Then setting

we have that

$$
\mathcal{H}_{1}=\left\{u \in H^{2}(M): \bar{u}=0, \quad \int_{M}\left|\Delta_{g} u\right|^{2} d V_{g}=1\right\}
$$

$$
\sup _{u \in \mathcal{H}_{1}} \int_{M} e^{32 \pi^{2} u^{2}} d V_{g}
$$

is attained.

On the 4-dimensional manifold $(M, g)$, the so-called Paneitz operator, which is defined in terms of the scalar curvature $R_{g}$ and the Ricci tensor Ric $_{g}$ as

$$
P_{g}^{4} u=\Delta_{g}^{2} u+\operatorname{div}_{g}\left(\frac{2}{3} R_{g} g-2 R i c_{g}\right) d u \quad u \in C^{\infty}(M),
$$

plays an important role in conformal geometry see [4], [6], [7], [8], [11], [20], [21]. In particular, the relation between the Paneitz operator and the $Q$-curvature, which is defined as

$$
Q_{g}=-\frac{1}{12}\left(\Delta_{g} R_{g}-R_{g}^{2}+3\left|R i c_{g}\right|^{2}\right),
$$

is of great interest. It is well-known that Moser-Trudinger inequalities involving $P_{g}^{4}$ play an important role in the problem of prescribing constant $Q$-curvature see [8], [12], [20]. Therefore it is worth having an extension of Theorem 1.1 concerning the Paneitz operator as well. Our next result goes in this direction. More precisely we have the following.

Theorem 1.2. Let $(M, g)$ be a compact closed smooth 4-dimensional Riemannian manifold. Assuming that $P_{g}^{4}$ is non-negative and $\operatorname{ker} P_{g}^{4} \simeq \mathbb{R}$, then setting

$$
\mathcal{H}_{2}=\left\{u \in H^{2}(M): \bar{u}=0, \quad<P_{g}^{4} u, u>=1\right\}
$$

we have

is attained.

$$
\sup _{u \in \mathcal{H}_{2}} \int_{M} e^{32 \pi^{2} u^{2}} d V_{g}
$$

Remark 1.3. Since the leading term of $P_{g}^{4}$ ( for the definition see the Section 2) is $\Delta_{g}^{2}$ then the two Theorems are quite similar. We point out that the same proof is valid for both except some trivial adaptations, hence we will give a full proof of Theorem 1.1 only and sketch the proof of Theorem 1.2 in the last section.

Remark 1.4. We mention that due to a result by Gursky, see [11] if both the Yamabe class $Y(g)$ and $\int_{M} Q_{g} d V_{g}$ are non-negative, then we have that $P_{g}^{4}$ is non-negative and $\operatorname{ker} P_{g}^{4} \simeq \mathbb{R}$.

We are going to describe our approach to prove Theorem 1.1. We will use Blow-up analysis. First of all we take a sequence $\left(\alpha_{k}\right)_{k}$ such that $\alpha_{k} \nearrow 32 \pi^{2}$, and by using Direct Methods of the Calculus of variations we can find $u_{k} \in \mathcal{H}_{1}$ such that

$$
\int_{M} e^{\alpha_{k} u_{k}^{2}} d V_{g}=\sup _{v \in \mathcal{H}_{1}} \int_{M} e^{\alpha_{k} v^{2}} d V_{g}
$$

see Lemma 3.1. Moreover using the Lagrange multiplier rule we have that $\left(u_{k}\right)_{k}$ satisfies the equation:

for some constants $\lambda_{k}$ and $\gamma_{k}$.

$$
\Delta_{g}^{2} u_{k}=\frac{u_{k}}{\lambda_{k}} e^{\alpha_{k} u_{k}^{2}}-\gamma_{k}
$$

Now it is easy to see that if there exists $\alpha>32 \pi^{2}$ such that $\int_{M} e^{\alpha u_{k}^{2}} d V_{g}$ is bounded, then by using Lagrange formula, Young's inequality and Rellich compactness Theorem, we obtain that the weak limit of $u_{k}$ becomes an extremizer. On the other hand if

$$
c_{k}=\max _{M}\left|u_{k}\right|=\left|u_{k}\right|\left(x_{k}\right)
$$


is bounded, then from standard elliptic regularity theory $u_{k}$ is compact, thus converges uniformly to an extremizer. Hence assuming that Theorem 1.1 does not hold, we get

1)

$$
\forall \alpha>32 \pi^{2} \quad \lim _{k \rightarrow+\infty} \int_{M} e^{\alpha u_{k}^{2}} d V_{g} \rightarrow+\infty
$$

$$
c_{k} \rightarrow+\infty
$$

We will follow the same method as in [14] up to some extents.

In [14], the function sequence we studied is the following:

$$
-\Delta_{g} u_{k}=\frac{u_{k}}{\lambda_{k}} e^{\alpha_{k}^{\prime} u_{k}^{2}}-\gamma_{k}
$$

where $\alpha_{k}^{\prime} \nearrow 4 \pi$, and $u_{k}$ attains $\sup _{\int_{M}\left|\nabla_{g} u\right|^{2} d V_{g}=1, \bar{u}=0} \int_{M} e^{\alpha_{k}^{\prime} u^{2}} d V_{g}$. We also assumed $c_{k} \rightarrow+\infty$. Then we have

$$
2 \alpha_{k} c_{k}\left(u_{k}\left(x_{k}+r_{k} x\right)-c_{k}\right) \rightarrow-2 \log \left(1+\pi|x|^{2}\right)
$$

for suitable choices of $r_{k}, x_{k}$. Next we proved the following

$$
\lim _{k \rightarrow+\infty} \int_{\left\{u_{k} \leq \frac{c_{k}}{A}\right\}}\left|\nabla_{g} u_{k}\right|^{2} d V_{g}=\frac{1}{A} \quad \forall A>1,
$$

which implies that

$$
\lim _{k \rightarrow+\infty} \int_{M} e^{\alpha_{k} u_{k}^{2}} d V_{g}=\mu(M)+\lim _{k \rightarrow+\infty} \frac{\lambda_{k}}{c_{k}^{2}},
$$

and that $c_{k} u_{k}$ converges to some Green function weakly. In the end, we got an upper bound of $\frac{\lambda_{k}}{c_{k}^{2}}$ via capacity.

Remark 1.5. (1.3) was first discovered by Struwe in [23].

Remark 1.6. (1.4) also appeared in [2].

However there are two main differences between the present case and the one in [14]. One is that there is no direct maximum principle for equation (1.2) and the other one is that truncations are not allowed in the space $H^{2}(M)$. Hence to get a counterpart of (1.3) and (1.4) is not easy.

To solve the first difficulty, we replace $c_{k}\left(u_{k}\left(x_{k}+r_{k} x\right)-c_{k}\right)$ with $\beta_{k}\left(u_{k}\left(\exp _{x_{k}}\left(r_{k} x\right)\right)-c_{k}\right)$, where

$$
1 / \beta_{k}=\int_{M} \frac{\left|u_{k}\right|}{\lambda_{k}} e^{\alpha_{k} u_{k}^{2}} d V_{g} .
$$

By using the strength of the Green representation formula, we get that the profile of $u_{k}$ is either a constant function or a standard bubble. The second difficulty will be solved by applying capacity and Pohozaev type identity. In more detail we will prove that $\beta_{k} u_{k} \rightarrow G$ (see Lemma 3.6) which satisfies

$$
\left\{\begin{array}{l}
\Delta_{g}^{2} G=\tau\left(\delta_{x_{0}}-\operatorname{Vol}_{g}(M)\right) \\
\int_{M} G=0
\end{array}\right.
$$

for some $\tau \in(0,1]$. Then we can derive from a Pohozaev type identity (see Lemma 3.7$)$ that

$$
\lim _{k \rightarrow+\infty} \int_{M} e^{\alpha_{k} u_{k}^{2}} d V_{g} \operatorname{Vol}_{g}(M)+\lim _{k \rightarrow+\infty} \tau^{2} \frac{\lambda_{k}}{\beta_{k}^{2}} .
$$

In order to apply the capacity, we will follow some ideas in [12]. Concretely, we will show that up to a small term the energy of $u_{k}$ on some annulus is bounded below by the Euclidean one (see Lemma 3.10). Moreover one can prove the existence of $U_{k}$ (see Lemma 3.11) such that the energy of $U_{k}$ is comparable to the Euclidean energy of $u_{k}$, and the Dirichlet datum and Neumann datum of $U_{k}$ at the boundary of the annulus are also comparable to those of $u_{k}$. In this sense, 
we simplify the calculation of capacity in [15]. Now using capacity techniques we get $\frac{c_{k}}{\beta_{k}} \rightarrow d$ and $d \tau=1$, see Proposition 3.12. Furthermore we have that

$$
\lim _{k \rightarrow+\infty} \tau^{2} \frac{\lambda_{k}}{\beta_{k}^{2}} \leq \frac{\pi^{2}}{6} e^{\frac{5}{3}+32 \pi^{2} S_{0}} .
$$

Hence we arrive to

$$
\sup _{u \in \mathcal{H}_{1}} \int_{M} e^{32 \pi^{2} u^{2}} d V_{g} \leq \operatorname{Vol}_{g}(M)+\frac{\pi^{2}}{6} e^{\frac{5}{3}+32 \pi^{2} S_{0}} .
$$

In the end, we will find test functions in order to contradict (1.5). We will simplify the arguments in [14]. Indeed we use carefully the regular part of $G$ to avoid cut-off functions and hence making the calculations simpler.

The plan of the paper is the following: In Section 2 we collect some preliminary results regarding the existence of the Green functions for $\Delta_{g}^{2}$ and $P_{g}^{4}$, and associated Moser-Trudinger type inequality. In Section 2 we prove Theorem 1.1. This Section is divided into six subsections. In the first one, we deal with concentration behavior and the profile of the blowing-up sequence. The second one is concerned about the derivation of a Pohozaev type identity and its application. In subsection 3 we perform the Blow-up analysis to get either the zero function or a standard bubble in the limit. In the subsection 4, we deal with the capacity estimates to get an upper bound. And in the subsection 5 , we construct test functions. In the last subsection we show how to reach a contradiction. The last Section is concerned about the sketch of the proof of Theorem 1.2.

\section{Acknowledgements}

The second author has been supported by M.U.R.S.T within the PRIN 2004 Variational methods and nonlinear differential equations.

\section{Notations and Preliminaries}

In this brief section we collect some useful notations, and state a lemma giving the existence of the Green functions of $\Delta_{g}^{2}$ and of the operator $P_{g}^{4}$ with the asymptotics near the singularity. We also give a version of Adams inequality on the a manifold due to Fontana and an analogue of the well-known Moser-Trudinger inequality for the operator $P_{g}^{4}$ when it is non-negative.

In the following, $B_{r}(x)$ stands for the metric ball of radius $r$ and center $x$ in $M, B^{r}(p)$ and stands for the Euclidean ball of center $p$ and radius $r$. We also denote with $d_{g}(x, y)$ the metric distance between two points $x$ and $y$ of $M . H^{2}(M)$ stands for the usual Sobolev space of functions on $M$, i.e functions which are in $L^{2}$ together with their first and second derivatives. $W^{2, q}(M)$ denotes the usual Sobolev spaces of functions which are in $L^{q}(M)$ with their first and second derivatives. Large positive constants are always denoted by $C$, and the value of $C$ is allowed to vary from formula to formula and also within the same line. $M^{2}$ stands for the cartesian product $M \times M$, while $\operatorname{Diag}(M)$ is the diagonal of $M^{2}$. Given a function $u \in L^{1}(M), \bar{u}$ denotes its average on $M$, that is $\bar{u}=\left(\operatorname{Vol}_{g}(M)\right)^{-1} \int_{M} u(x) d V_{g}(x)$ where $\operatorname{Vol}_{g}(M)=\int_{M} d V_{g}$.

$A_{k}=o_{k}(1)$ means that $A_{k} \rightarrow 0$ as the integer $k \rightarrow+\infty$.

$A_{\delta}=o_{\delta}(1)$ means that $A_{\delta} \rightarrow 0$ as the real number $\delta \longrightarrow 0$.

$A_{k, \delta}=o_{k, \delta}(1)$ means that $A_{k, \delta} \rightarrow 0$ as $k \rightarrow+\infty$ first and after the real number $\delta \longrightarrow 0$.

$A_{k}=O\left(B_{k}\right)$ means that $A_{k} \leq C B_{k}$ for some fixed constant $C$.

$\operatorname{inj}_{g}(M)$ stands for the injectivity radius of $M$.

$d V_{g}$ denotes the Riemannian measure associated to the metric $g$.

$d S_{g}$ stands for the surface measure associated to $g$.

Given a metric $g$ on $M$, and $x \in M,|g(x)|$, stands for determinant of the matrix with entries $g_{i, j}(x)$ where $g_{i, j}(x)$ are the components of $g(x)$ in some system of coordinates.

$\Delta_{0}$ stands for the Euclidean Laplacian and $\Delta_{g}$ the Laplace-Beltrami with respect to the background metric $g$.

As mentioned before we begin by stating a lemma giving the existence of the Green function of $\Delta_{g}^{2}$ and $P_{g}^{4}$, and their asymptotics near the singularities. 
Lemma 2.1. We have that the Green function $F(x, y)$ of $\Delta_{g}^{2}$ exists in the following sense:

a) For all functions $u \in C^{2}(M)$, we have

$$
u(x)-\bar{u}=\int_{M} F(x, y) \Delta_{g}^{2} u(y) d V_{g}(y) \quad x \neq y \in M
$$

b)

$$
F(x, y)=H(x, y)+K(x, y)
$$

is smooth on $M^{2} \backslash \operatorname{Diag}\left(M^{2}\right), K$ extends to a $C^{1+\alpha}$ function on $M^{2}$ and

$$
H(x, y)=\frac{1}{8 \pi^{2}} f(r) \log \frac{1}{r}
$$

where, $r=d_{g}(x, y)$ is the geodesic distance from $x$ to $y ; f(r)$ is a $C^{\infty}$ positive decreasing function, $f(r)=1$ in a neighborhood of $r=0$ and $f(r)=0$ for $r \geq i n j_{g}(M)$. Moreover we have that the following estimates holds

$$
\left|\nabla_{g} F(x, y)\right| \leq C \frac{1}{d_{g}(x, y)} \quad\left|\nabla_{g}^{2} F(x, y)\right| \leq C \frac{1}{d_{g}(x, y)^{2}} .
$$

Proof. For the proof see [6] and the proof of Lemma 2.3 in [17].

Lemma 2.2. Suppose $K$ er $P_{g}^{4} \simeq \mathbb{R}$. Then the Green function $Q(x, y)$ of $P_{g}^{4}$ exists in the following sense:

a) For all functions $u \in C^{2}(M)$, we have

$$
u(x)-\bar{u}=\int_{M} Q(x, y) P_{g}^{4} u(y) d V_{g}(y) \quad x \neq y \in M
$$

b)

$$
Q(x, y)=H_{0}(x, y)+K_{0}(x, y)
$$

is smooth on $M^{2} \backslash \operatorname{Diag}\left(M^{2}\right), K$ extends to a $C^{2+\alpha}$ function on $M^{2}$ and

$$
H(x, y)=\frac{1}{8 \pi^{2}} f(r) \log \frac{1}{r}
$$

where, $r=d_{g}(x, y)$ is the geodesic distance from $x$ to $y ; f(r)$ is a $C^{\infty}$ positive decreasing function, $f(r)=1$ in a neighborhood of $r=0$ and $f(r)=0$ for $r \geq i n j_{g}(M)$.

Proof. For the proof see Lemma 2.1 in [20].

Next we state a Theorem due to Fontana[9].

Theorem 2.3. ([9]) There exists a constant $C=C(M)>0$ such that the following holds

$$
\int_{M} e^{32 \pi^{2} u^{2}} d V_{g} \leq C \text { for all } u \in H^{2}(M) \text { such that } \int_{M}\left|\Delta_{g}^{2} u\right| d V_{g}=1 \text {. }
$$

Moreover this constant is optimal in the sense that if we replace it by any $\alpha$ bigger then the integral can be maken as large as we want.

Next we state a Moser-Trudinger type inequality corresponding to $P_{g}^{4}$ when it is non-negative. The proof can be found in [20] where it is proven for every $P_{g}^{n}$ (where $P_{g}^{n}$ stands for higher order Paneitz operator).

Proposition 2.4. Suppose that $P_{g}^{4}$ is non-negative and that $k e r P_{g}^{4}=\mathbb{R}$, then there exists a constant $C=C(M)>0$ such that

$$
\int_{M} e^{32 \pi^{2} u^{2}} d V_{g} \leq C \text { for all } u \in H^{2}(M) \text { such that }\left\langle P_{g}^{4} u, u\right\rangle=1 \text {. }
$$




\section{Proof of Theorem 1.1}

Lemma 3.1. Let $\alpha_{k}$ be an increasing sequence converging to $32 \pi^{2}$. Then for every $k$ there exists $u_{k} \in \mathcal{H}_{1}$ such that

$$
\int_{M} e^{\alpha_{k} u_{k}^{2}} d V_{g}=\sup _{u \in \mathcal{H}_{1}} \int_{M} e^{\alpha_{k} u^{2}} d V_{g} .
$$

Moreover $u_{k}$ satisfies the following equation

$$
\Delta_{g}^{2} u_{k}=\frac{1}{\lambda_{k}} u_{k} e^{\alpha_{k} u_{k}^{2}}-\gamma_{k}
$$

where

$$
\lambda_{k}=\int_{M} u_{k}^{2} e^{\alpha_{k} u_{k}^{2}} d V_{g}
$$

and

$$
\gamma_{k}=\frac{1}{\lambda_{k} V o l_{g}(M)} \int_{M} u_{k} e^{\alpha_{k} u_{k}^{2}} d V_{g} .
$$

Moreover we have $u_{k} \in C^{\infty}(M)$.

Proof. First of all using the inequality in Theorem 2.3, one can check easily that the functional

$$
I_{k}(u)=\int_{M} e^{\alpha_{k} u^{2}} d V_{g}
$$

is weakly continous. Hence using Direct Methods of the Calculus of Variations we get the existence of maximizer say $u_{k}$. On the other hand using the Lagrange multiplier rule one get the equation (3.1). Moreover integrating the equation (3.1) and after multipling it by $u_{k}$ and integrating again, we get the value of $\gamma_{k}$ and $\lambda_{k}$ respectively. Moreover using standard elliptic regularity we get that $u_{k} \in C^{\infty}(M)$. Hence the Lemma is proved.

Now we are ready to give the proof of Theorem 1.1. ¿From now on we suppose by contradiction that Theorem 1.1 does not hold. Hence from the same considerations as in the Introduction we have that :

1)

$$
\forall \alpha>32 \pi^{2} \lim _{k \rightarrow+\infty} \int_{M} e^{\alpha u_{k}^{2}} d V_{g} \rightarrow+\infty
$$

2)

$$
c_{k}=\max _{M}\left|u_{k}\right|=\left|u_{k}\right|\left(x_{k}\right) \rightarrow+\infty
$$

We will divide the reminder of the proof into six subsections.

3.1. Concentration behavior and profile of $u_{k}$. This subsection is concerned about two main ingredients. The first one is the study of the concentration phenomenon of the energy corresponding to $u_{k}$. The second one is the description of the profile of $\beta_{k} u_{k}$ as $k \rightarrow+\infty$, where $\beta_{k}$ is given by the relation

$$
1 / \beta_{k}=\int_{M} \frac{\left|u_{k}\right|}{\lambda_{k}} e^{\alpha_{k} u_{k}^{2}} d V_{g}
$$

We start by giving an energy concentration lemma which is inspired from P.L.Lions'work.

Lemma 3.2. $u_{k}$ verifies :

$$
u_{k} \rightarrow 0 \text { in } H^{2}(M) \text {; }
$$

and

$$
\left|\Delta_{g} u_{k}\right|^{2} \rightarrow \delta_{x_{0}}
$$

for some $x_{0} \in M$. 
Proof. First of all from the fact that $u_{k} \in \mathcal{H}_{1}$ we can assume without loss of generality that

$$
u_{k} \rightarrow u_{0} \text { in } H^{2}(M)
$$

Now let us show that $u_{0}=0$.

We have the trivial identity

$$
\int_{M}\left|\Delta_{g}\left(u_{k}-u_{0}\right)\right|^{2} d V_{g}=\int_{M}\left|\Delta_{g} u_{k}\right|^{2} d V_{g}+\int_{M}\left|\Delta_{g} u_{0}\right|^{2} d V_{g}-2 \int_{M} \Delta_{g} u_{k} \Delta_{g} u_{0} d V_{g} .
$$

Hence using the fact that $\int_{M}\left|\Delta_{g} u_{k}\right|^{2} d V_{g}=1$ we derive

$$
\int_{M}\left|\Delta_{g}\left(u_{k}-u_{0}\right)\right|^{2} d V_{g}=1+\int_{M}\left|\Delta_{g} u_{0}\right|^{2} d V_{g}-2 \int_{M} \Delta_{g} u_{k} \Delta_{g} u_{0} d V_{g}
$$

So using (3.3) we get

$$
\lim _{k \rightarrow 0} \int_{M}\left|\Delta_{g}\left(u_{k}-u_{0}\right)\right|^{2} d V_{g} 1-\int_{M} \Delta_{g} u_{0} \Delta_{g} u_{0} d V_{g}
$$

Now suppose that $u_{0} \neq 0$ and let us argue for a contradiction. Then there exists some $\beta<1$ such that for $k$ large enough the following holds

$$
\int_{M}\left|\Delta_{g}\left(u_{k}-u_{0}\right)\right|^{2} d V_{g}<\beta
$$

Hence using Fontana's result see Theorem 2.3 we obtain that

$$
\int_{M} e^{\alpha_{1}\left(u_{k}-u_{0}\right)^{2}} d V_{g} \leq C \text { for some } \alpha_{1}>32 \pi^{2} .
$$

Now using Cauchy inequality one can check easily that

$$
\int_{M} e^{\alpha_{2} u_{k}^{2}} d V_{g} \leq C \text { for some } \alpha_{2}>32 \pi^{2} .
$$

Hence reaching a contradiction to (3.2).

On the other hand without lost of generality we can assume that

$$
\left|\Delta_{g} u_{k}\right| d V_{g} \rightarrow \mu
$$

Now suppose $\mu \neq \delta_{p}$ for every $p \in M$ and let us argue for a contradiction to (3.2) again. First of all let us take a cut-off function $\eta \in C_{0}^{\infty}\left(B_{\delta}(x)\right), \eta=1$ on $B_{x}\left(\frac{\delta}{2}\right)$ where $x$ is a fixed point in $M$ and $\delta$ a fixed positive and small number.

We have that

$$
\limsup _{k \rightarrow+\infty} \int_{B_{\delta}(x)}\left|\Delta_{g} u_{k}\right|^{2} d V_{g}<1
$$

Now working in a normal coordinate system around $x$ and using standard elliptic regularity theory we get

$$
\int_{B^{\delta}(\tilde{x})}\left|\Delta_{0} \widetilde{\eta u_{k}}\right|^{2} d V_{g} \leq\left(1+o_{\delta}(1)\right) \int_{B_{\delta}(x)}\left|\Delta_{g} u_{k}\right|^{2} d V_{g}
$$

where $\tilde{x}$ is the point corresponding to $x$ in $\mathbb{R}^{4}$ and $\widetilde{\eta u_{k}}$ the expression of $\eta u_{k}$ on the normal coordinate system. Hence for $\delta$ small we get

$$
\int_{B^{\delta}(\tilde{x})}\left|\Delta_{0} \widetilde{\eta u_{k}}\right|^{2} d V_{g}<1
$$

Thus using the Adams result see [1] we have that

$$
\int_{B^{\delta}(\tilde{x})} e^{\tilde{\alpha}\left(\widetilde{\left.\eta u_{k}\right)^{2}}\right.} d x \leq C \text { for some } \tilde{\alpha}>32 \pi^{2} .
$$

Hence using a covering argument we infer that

$$
\int_{M} e^{\bar{\alpha} u_{k}^{2}} d V_{g} \leq C \text { for some } \bar{\alpha}>32 \pi^{2},
$$

so reaching a contradiction. Hence the Lemma is proved. 
Lemma 3.3. We have the following hold:

$$
\lim _{k \rightarrow+\infty} \lambda_{k}=+\infty, \quad \lim _{k \rightarrow+\infty} \gamma_{k}=0
$$

Proof. Let $N>0$ be large enougth. By using the definition of $\lambda_{k}$ we have that

$$
\lambda_{k}=\int_{M} u_{k}^{2} e^{\alpha_{k} u_{k}^{2}} d V_{g} \geq N^{2} \int_{\left\{u_{k} \geq N\right\}} e^{\alpha_{k} u_{k}^{2}} d V_{g}=N^{2}\left(\int_{M} e^{\alpha_{k} u_{k}^{2}} d V_{g}-\int_{\left\{u_{k} \leq N\right\}} e^{\alpha_{k} u_{k}^{2}} d V_{g}\right) .
$$

On the other hand

$$
\lim _{k \rightarrow+\infty}\left(\int_{M} e^{\alpha_{k} u_{k}^{2}} d V_{g}-\int_{\left\{u_{k} \leq N\right\}} e^{\alpha_{k} u_{k}^{2}} d V_{g}\right)=\lim _{k \rightarrow+\infty} \int_{M} e^{\alpha_{k} u_{k}^{2}} d V_{g}-\operatorname{Vol}_{g}(M) .
$$

Hence using the fact that

$$
\lim _{k \rightarrow+\infty} \int_{M} e^{\alpha_{k} u_{k}^{2}} d V_{g}=\sup _{u \in \mathcal{H}_{1}} \int_{M} e^{32 \pi^{2} u^{2}} d V_{g}>\operatorname{Vol}_{g}(M)
$$

we have that 1) holds. Now we prove 2). using the definition of $\gamma_{k}$, we get

$$
\left|\gamma_{k}\right| \leq \frac{N}{\lambda_{k}} N e^{32 \pi^{2} N^{2}}+\frac{1}{V o l_{g}(M)} \frac{1}{N} .
$$

Hence by using point 1 and letting $k \rightarrow+\infty$ and after $N \rightarrow+\infty$ we get point 2 . So the Lemma is proved.

Next let us set

$$
\tau_{k}=\int_{M} \frac{\beta_{k} u_{k}}{\lambda_{k}} e^{\alpha_{k} u_{k}^{2}}
$$

One can check easily the following

Lemma 3.4. With the definition above we have that $0 \leq \beta_{k} \leq c_{k},\left|\tau_{k}\right| \leq 1$ and $\beta_{k} \gamma_{k}$ is bounded. Moreover up to a subsequence and up to changing $u_{k}$ to $-u_{k}$

$$
\tau_{k} \rightarrow \tau \geq 0 .
$$

The next Lemma gives some Lebesgue estimates on Ball in terms of the radius with constant independent of the ball. As a corollary we get the profile of $\beta_{k} u_{k}$ as $k \rightarrow+\infty$.

Lemma 3.5. There are constants $C_{1}(p)$, and $C_{2}(p)$ depending only on $p$ and $M$ such that, for $r$ sufficiently small and for any $x \in M$ there holds

$$
\int_{B_{r}(x)}\left|\nabla_{g}^{2} \beta_{k} u_{k}\right|^{p} d V_{g} \leq C_{2}(p) r^{4-2 p}
$$

and

$$
\int_{B_{r}(x)}\left|\nabla_{g} \beta_{k} u_{k}\right|^{p} d V_{g} \leq C_{1}(p) r^{4-p}
$$

where, respectively, $p<2$, and $p<4$.

Proof. First of all using the Green representation formula we have

$$
u_{k}(x)=\int_{M} F(x, y) \Delta_{g}^{2} u_{k} d V_{g}(y) \quad \forall x \in M .
$$

Hence using the equation we get

$$
u_{k}(x)=\int_{M} F(x, y)\left(\frac{1}{\lambda_{k}} u_{k} e^{\alpha_{k} u_{k}^{2}}\right) d V_{g}(y)-\int_{M} F(x, y) \gamma_{k} d V_{g}(y) .
$$

Now by differentiating with respect to $x$ for every $m=1,2$ we have that

$$
\left|\nabla_{g}^{m} u_{k}(x)\right| \leq \int_{M}\left|\nabla_{g}^{m} F(x, y)\right|\left(\frac{1}{\lambda_{k}}\right)\left|u_{k}\right| e^{\alpha_{k} u_{k}^{2}} d V_{g}(y)+\int_{M}\left|\nabla_{g}^{m} F(x, y)\right|\left|\gamma_{k}\right| .
$$

Hence we get

$$
\left|\nabla_{g}^{m}\left(\beta_{k} u_{k}(x)\right)\right| \leq \int_{M}\left|\nabla_{g}^{m} F(x, y)\right| \beta_{k}\left(\frac{1}{\lambda_{k}}\right)\left|u_{k}\right| e^{\alpha_{k} u_{k}^{2}} d V_{g}(y)+\int_{M}\left|\nabla_{g}^{m} F(x, y)\right| \beta_{k}\left|\gamma_{k}\right| .
$$


Taking the $p$-th power in both side of the inequality and using the basic inequality

$$
(a+b)^{p} \leq 2^{p-1}\left(a^{p}+b^{p}\right) \text { for } a \geq 0 \text { and } b \geq 0
$$

we obtain

$$
\begin{aligned}
\left|\nabla_{g}^{m}\left(\beta_{k} u_{k}(x)\right)\right|^{p} \leq 2^{p-1}\left[\int_{M}\left|\nabla_{g}^{m} F(x, y)\right| \beta_{m}\left(\frac{1}{\lambda_{k}}\right)\left|u_{k}\right| e^{\alpha_{k} u_{k}^{2}} d V_{g}(y)\right]^{p} \\
+2^{p-1}\left[\int_{M}\left|\nabla_{g}^{m} F(x, y)\right| \beta_{k}\left|\gamma_{k}\right|\right]^{p}
\end{aligned}
$$

Now integrating both sides of the inequality we obtain

$$
\begin{array}{r}
\int_{B_{r}(x)}\left|\nabla_{g}^{m}\left(\beta_{k} u_{k}(z)\right)\right| d V_{g}(z) \leq 2^{p-1} \int_{B_{r}(x)}\left[\int_{M}\left|\nabla_{g}^{m} F(z, y)\right| \beta_{k}\left(\frac{1}{\lambda_{k}}\right)\left|u_{k}\right| e^{\alpha_{k} u_{k}^{2}} d V_{g}(y)\right]^{p} d V_{g}(z) \\
+2^{p-1} \int_{B_{r}(x)}\left[\int_{M}\left|\nabla_{g}^{m} F(z, y)\right| \beta_{k}\left|\gamma_{k}\right|\right]^{p} d V_{g}(z) .
\end{array}
$$

First let us estimate the second term in the right hand side of the inequality

$$
\int_{B_{r}(x)}\left[\int_{M}\left|\nabla_{g}^{m} F(z, y)\right| \beta_{k}\left|\gamma_{k}\right|\right]^{p} d V_{g}(z) \leq C \int_{B_{r}(x)} \sup _{y \in M} \frac{1}{d_{g}(z, y)^{p m}} d V_{g}(z) \leq C(M) r^{4-m p}
$$

Thanks to the fact that $\beta_{k} \gamma_{k}$ is bounded, to the asymptotics of the Green function and to Jensen's inequality. Now let us estimates the second term. First of all we define the following auxiliary measure

$$
m_{k}=\beta_{k}\left(\frac{1}{\lambda_{k}}\right)\left|u_{k}\right| e^{\alpha_{k} u_{k}^{2}} d V_{g}
$$

We have that $m_{k}$ is a probability measure. On the other hand we can write

$$
\begin{array}{r}
\int_{B_{r}(x)}\left[\int_{M}\left|\nabla_{g}^{m} F(z, y)\right| \beta_{k}\left(\frac{1}{\lambda_{k}}\right)\left|u_{k}\right| e^{\alpha_{k} u_{k}^{2}} d V_{g}(y)\right]^{p} d V_{g}(z) \\
=\int_{B_{r}(x)}\left[\int_{M}\left|\nabla_{g}^{m} F(z, y)\right| d m_{k}(y)\right]^{p} d V_{g}(z) .
\end{array}
$$

Now by using Jensen's inequality we have that

$$
\left[\int_{M}\left|\nabla_{g}^{m} F(z, y)\right| d m_{k}(y)\right]^{p} \leq\left[\int_{M}\left|\nabla_{g}^{m} F(z, y)\right|^{p} d m_{k}(y)\right]
$$

Thus with the (3.4) we have that

$$
\begin{array}{r}
\int_{B_{r}(x)}\left[\int_{M}\left|\nabla_{g}^{m} F(z, y)\right| \beta_{k}\left(\frac{1}{\lambda_{k}}\right)\left|u_{k}\right| e^{\alpha_{k} u_{k}^{2}} d V_{g}(y)\right]^{p} d V_{g}(z) \leq \\
\int_{B_{r}(x)}\left[\int_{M}\left|\nabla_{g}^{m} F(z, y)\right|^{p} d m_{k}(y)\right] d V_{g}(z) .
\end{array}
$$

Now by using again the same argument as in the first term we obtain

$$
\int_{B_{r}(x)}\left[\int_{M}\left|\nabla_{g}^{m} F(z, y)\right|^{p} d m_{k}(y)\right] d V_{g}(z) \leq C(M) r^{4-m p} .
$$

Hence the Lemma is proved.

Next we give a corollary of this Lemma.

Corollary 3.6. We have $\beta_{k} u_{k} \rightarrow G W^{2, p}(M)$ for $p \in(1,2), \beta_{k} u_{k} \rightarrow G$ smoothly in $M \backslash B_{\delta}\left(x_{0}\right)$ where $\delta$ is small and $G$ satisfies

$$
\left\{\begin{array}{l}
\Delta_{g}^{2} G=\tau\left(\delta_{x_{0}}-\frac{1}{V o l_{g}(M)}\right) \text { in } M \\
\bar{G}=0
\end{array}\right.
$$

Moreover

$$
G(x)=\frac{\tau}{8 \pi^{2}} \log \frac{1}{r}+\tau S(x)
$$


with $r=d_{g}\left(x, x_{0}\right) . S=S_{0}+S_{1}(x), S_{0}=S\left(x_{0}\right)$ and $S \in W^{2, q}(M)$ for every $q \geq 1$.

Proof. By Lemma 3.5 we have that

$$
\beta_{k} u_{k} \rightarrow G W^{2, p}(M) \quad p \in(1,2)
$$

On the other hand using Lemma 3.2 we get $e^{\alpha_{k} u_{k}^{2}}$ is bounded in $L^{p}\left(M \backslash B_{\delta}\left(x_{0}\right)\right)$. Hence the standard elliptic regularity implies that

$$
\beta_{k} u_{k} \rightarrow G \text { smoothly in } M \backslash B_{\delta}\left(x_{0}\right) .
$$

So to end the proof of the proposition we need only to show that

$$
\frac{\beta_{k}}{\lambda_{k}} u_{k} e^{\alpha_{k} u_{k}^{2}} \rightarrow \tau \delta_{x_{0}}
$$

To do this let us take $\varphi \in C^{\infty}(M)$ then we have

$$
\int_{M} \varphi \frac{\beta_{k}}{\lambda_{k}} u_{k} e^{\alpha_{k} u_{k}^{2}} d V_{g}=\int_{M \backslash B_{\delta}\left(x_{0}\right)} \varphi \frac{\beta_{k}}{\lambda_{k}} u_{k} e^{\alpha_{k} u_{k}^{2}} d V_{g}+\int_{B_{\delta}\left(x_{0}\right)} \varphi \frac{\beta_{k}}{\lambda_{k}} u_{k} e^{\alpha_{k} u_{k}^{2}} d V_{g}
$$

Using (3.5) we have that

$$
\int_{M \backslash B_{\delta}\left(x_{0}\right)} \varphi \frac{\beta_{k}}{\lambda_{k}} u_{k} e^{\alpha_{k} u_{k}^{2}} d V_{g}=O\left(\frac{1}{\lambda_{k}}\right) .
$$

On the other hand, we can write inside the ball $B_{\delta}\left(x_{0}\right)$

$$
\begin{aligned}
\int_{B_{\delta}\left(x_{0}\right)} \varphi \frac{\beta_{k}}{\lambda_{k}} u_{k} e^{\alpha_{k} u_{k}^{2}} d V_{g} & =\left(\varphi\left(x_{0}\right)+o_{\delta}(1)\right) \int_{B_{\delta}\left(x_{0}\right)} \frac{\beta_{k}}{\lambda_{k}} u_{k} e^{\alpha_{k} u_{k}^{2}} d V_{g} \\
& =\left(\varphi\left(x_{0}\right)+o_{\delta}(1)\right)\left(\tau-\int_{M \backslash B_{\delta}\left(x_{0}\right)} \frac{\beta_{k}}{\lambda_{k}} u_{k} e^{\alpha_{k} u_{k}^{2}} d V_{g}\right)
\end{aligned}
$$

Now using again (3.5) we derive

$$
\int_{M \backslash B_{\delta}\left(x_{0}\right)} \frac{\beta_{k}}{\lambda_{k}} u_{k} e^{\alpha_{k} u_{k}^{2}}=O\left(\frac{1}{\lambda_{k}}\right) .
$$

Hence we arrive to

$$
\int_{B_{\delta}\left(x_{0}\right)} \varphi \frac{\beta_{k}}{\lambda_{k}} u_{k} e^{\alpha_{k} u_{k}^{2}} d V_{g}=\tau \varphi\left(x_{0}\right)+o_{k, \delta}(1)
$$

Thus we get

$$
\int_{M} \varphi \frac{\beta_{k}}{\lambda_{k}} u_{k} e^{\alpha_{k} u_{k}^{2}} d V_{g}=O\left(\frac{1}{\lambda_{k}}\right)+\tau \varphi\left(x_{0}\right)+o_{k, \delta}(1) .
$$

Hence from Lemma 3.3 we conclude the proof of claim (3.6) )and of the Corollary too.

3.2. Pohozaev type identity and application. As it is already said in the introduction this subsection deals with the derivation of a Pohozaev type identity. And as corollary we give the limit of $\int_{M} e^{\alpha_{k} u_{k}^{2}} d V_{g}$ in terms of $\operatorname{Vol}_{g}(M), \lambda_{k}, \beta_{k}$ and $\tau$

Lemma 3.7. Setting $U_{k}=\Delta_{g} u_{k}$ we have the following holds

$$
\begin{array}{r}
-\frac{2}{\alpha_{k} \lambda_{k}} \int_{B_{\delta}\left(x_{k}\right)} e^{\alpha_{k} u_{k}^{2}} d V_{g}=-\frac{\delta}{2} \int_{\partial B_{\delta}\left(x_{k}\right)} U_{k}^{2} d S_{g}-\delta \int_{\partial B_{\delta}\left(x_{k}\right)} \nabla_{g} u_{k} \nabla_{g} U_{k} d V_{g}+2 \int_{\partial B_{\delta}\left(x_{k}\right)} U_{k} \frac{\partial u_{k}}{\partial r} \\
+2 \delta \int_{\partial B_{\delta}\left(x_{k}\right)} \frac{\partial U_{k}}{\partial r} \frac{\partial u_{k}}{\partial r} d S_{g}+\int_{B_{\delta}\left(x_{k}\right)} O\left(r^{2}\right) \nabla_{g} u_{k} \nabla_{g} U_{k} d V_{g} \\
\quad+\int_{B_{\delta}\left(x_{k}\right)} O\left(r^{2}\right) U_{k}^{2} d V_{g}+\int_{B_{\delta}\left(x_{k}\right)} e^{\alpha_{k} u_{k}^{2}} O\left(r^{2}\right) d V_{g}-\frac{\delta}{2 \lambda_{k} \alpha_{k}} \int_{\partial B_{\delta}\left(x_{k}\right)} e^{\alpha_{k} u_{k}^{2}} d V_{g}+O\left(\frac{\delta}{\beta_{k}^{2}}\right) .
\end{array}
$$

where $\delta$ is small and fixed real number. 
Proof. The proof relies on the divergence formula and the asymptotics of the metric $\mathrm{g}$ in normal coordinates around $x_{k}$.

By the definition of $U_{k}$ we have that

$$
\left\{\begin{array}{l}
\Delta_{g} u_{k}=U_{k} \\
\Delta_{g} U_{k}=\frac{u_{k}}{\lambda_{k}} e^{\alpha_{k} u_{k}^{2}}-\gamma_{k} .
\end{array}\right.
$$

The first issue is to compute $\int_{B_{\delta}\left(x_{k}\right)} r \frac{\partial U_{k}}{\partial r} \Delta_{g} u_{k}$ in two different ways, where $r(x)=d_{g}\left(x, x_{k}\right)$. On one side we obtain

$$
\int_{B_{\delta}\left(x_{k}\right)} r \frac{\partial U_{k}}{\partial r} \Delta_{g} u_{k} d V_{g}=-\int_{B_{\delta}\left(x_{k}\right)}\left(\nabla_{g} U_{k} \nabla_{g} u_{k}+r \frac{\partial \nabla_{g} U_{k}}{\partial r} \nabla_{g} u_{k}\right) d V_{g}+\int_{\partial B_{\delta}\left(x_{k}\right)} r \frac{\partial U_{k}}{\partial r} \frac{\partial u_{k}}{\partial r} d S_{g} .
$$

On the other side we get

$$
\begin{aligned}
\int_{B_{\delta}\left(x_{k}\right)} r \frac{\partial U_{k}}{\partial r} \Delta_{g} u_{k} d V_{g} & =\int_{B_{\delta}\left(x_{k}\right)} r \frac{\partial U_{k}}{\partial r} U_{k} d V_{g} \\
& =\int_{0}^{\delta} 2 \pi^{2} \int_{\partial B_{r}\left(x_{k}\right)} \frac{\partial U_{k}}{\partial r} U_{k} \sqrt{|g|} r^{4} d S d r \\
& =\frac{\delta}{2} \int_{\partial B_{\delta}\left(x_{k}\right)} U_{k}^{2} d S_{g}-2 \int_{B_{\delta}\left(x_{k}\right)} U_{k}^{2}\left(1+O\left(r^{2}\right)\right) d V_{g} .
\end{aligned}
$$

Thus we have

$$
\begin{aligned}
& \frac{\delta}{2} \int_{\partial B_{\delta}\left(x_{k}\right)} U_{k}^{2} d S_{g}-2 \int_{B_{\delta}\left(x_{k}\right)} U_{k}^{2} d V_{g}=-\int_{B_{\delta}\left(x_{k}\right)}\left(\nabla_{g} U_{k} \nabla_{g} u_{k}+r \frac{\partial \nabla_{g} U_{k}}{\partial r} \nabla_{g} u_{k}\right) d V_{g} \\
& +\int_{\partial B_{\delta}\left(x_{k}\right)} r \frac{\partial U_{k}}{\partial r} \frac{\partial v_{k}}{\partial r} d S_{g}+\int_{B_{\delta}\left(x_{k}\right)} O\left(r^{2}\right) U_{k}^{2} d V_{g}
\end{aligned}
$$

In the same way we obtain

$$
\begin{aligned}
& \frac{\delta}{2 \lambda_{k} \alpha_{k}} \int_{\partial B_{\delta}\left(x_{k}\right)} e^{\alpha_{k} u_{k}^{2}} d S_{g}-\frac{2}{\lambda_{k} \alpha_{k}} \int_{B_{\delta}\left(x_{k}\right)} e^{\alpha_{k} u_{k}^{2}}\left(1+O\left(r^{2}\right)\right) d V_{g} \\
& \quad=-\int_{B_{\delta}\left(x_{k}\right)}\left(\nabla_{g} U_{k} \nabla_{g} u_{k}+r \frac{\partial \nabla_{g} u_{k}}{\partial r} \nabla_{g} U_{k}\right) d V_{g}+\int_{\partial B_{\delta}\left(x_{k}\right)} r \frac{\partial U_{k}}{\partial r} \frac{\partial u_{k}}{\partial r} d S_{g}+O\left(\frac{\delta}{\beta_{k}^{2}}\right) .
\end{aligned}
$$

Hence by summing this two last lines we arrive to

$$
\begin{aligned}
\frac{\delta}{2 \lambda_{k} \alpha_{k}} & \int_{\partial B_{\delta}\left(x_{k}\right)} e^{\alpha_{k} u_{k}^{2}} d S_{g}-\frac{2}{\lambda_{k} \alpha_{k}} \int_{B_{\delta}\left(x_{k}\right)} e^{\alpha_{k} u_{k}^{2}} d V_{g}+\frac{\delta}{2} \int_{\partial B_{\delta}\left(x_{k}\right)} U_{k}^{2} d S_{g}-2 \int_{B_{\delta}\left(x_{k}\right)} U_{k}^{2} d V_{g} \\
= & -\int_{B_{\delta}\left(x_{k}\right)}\left(2 \nabla_{g} U_{k} \nabla_{g} u_{k}+r \frac{\partial}{\partial r} \nabla_{g} u_{k} \nabla_{g} U_{k}\right) d V_{g}+2 \int_{\partial B_{\delta}\left(x_{k}\right)} r \frac{\partial U_{k}}{\partial r} \frac{\partial u_{k}}{\partial r} d S_{g} \\
& +\int_{B_{\delta}\left(x_{k}\right)} O\left(r^{2}\right) U_{k}^{2} d V_{g}+\int_{B_{\delta}\left(x_{k}\right)} e^{\alpha_{k} u_{k}^{2}} O\left(r^{2}\right) d V_{g}+O\left(\frac{\delta}{\beta_{k}^{2}}\right) .
\end{aligned}
$$

On the other hand using the same method one can check easily that

$$
\begin{aligned}
\int_{B_{\delta}\left(x_{k}\right)} r \frac{\partial}{\partial r} \nabla_{g} u_{k} \nabla_{g} U_{k} d V_{g}= & \delta \int_{\partial B_{\delta}\left(x_{k}\right)} \nabla_{g} u_{k} \nabla_{g} U_{k} d V_{g}-4 \int_{B_{\delta}\left(x_{k}\right)} \nabla_{g} u_{k} \nabla_{g} U_{k} d V_{g} \\
& +\int_{B_{\delta}\left(x_{k}\right)} O\left(r^{2}\right) \nabla_{g} u_{k} \nabla_{g} U_{k} d V_{g}
\end{aligned}
$$

and

$$
\begin{aligned}
\int_{B_{\delta}\left(x_{k}\right)} \nabla_{g} U_{k} \nabla_{g} u_{k} d V_{g} & =-\int_{B_{\delta}\left(x_{k}\right)} U_{k} \Delta_{g} u_{k} d V_{g}+\int_{\partial B_{\delta}\left(x_{k}\right)} U_{k} \frac{\partial u_{k}}{\partial r} d S_{g} \\
& =-\int_{B_{\delta}\left(x_{k}\right)} U_{k}^{2} d V_{g}+\int_{\partial B_{\delta}\left(x_{k}\right)} U_{k} \frac{\partial u_{k}}{\partial r} d S_{g}
\end{aligned}
$$


So using (3.7),(3.8) and (3.9) we arrive to

$$
\begin{aligned}
-\frac{2}{\alpha_{k} \lambda_{k}} \int_{B_{\delta}\left(x_{k}\right)} e^{\alpha_{k} u_{k}^{2}} d V_{g}= & -\frac{\delta}{2} \int_{\partial B_{\delta}\left(x_{k}\right)} U_{k}^{2} d S_{g}-\delta \int_{\partial B_{\delta}\left(x_{k}\right)} \nabla_{g} u_{k} \nabla_{g} U_{k} d V_{g}+2 \int_{\partial B_{\delta}\left(x_{k}\right)} U_{k} \frac{\partial u_{k}}{\partial r} \\
& +2 \delta \int_{\partial B_{\delta}\left(x_{k}\right)} \frac{\partial U_{k}}{\partial r} \frac{\partial u_{k}}{\partial r} d S_{g}+\int_{B_{\delta}\left(x_{k}\right)} O\left(r^{2}\right) \nabla_{g} u_{k} \nabla_{g} U_{k} d V_{g} \\
& +\int_{B_{\delta}\left(x_{k}\right)} O\left(r^{2}\right) U_{k}^{2} d V_{g}+\int_{B_{\delta}\left(x_{k}\right)} e^{\alpha_{k} u_{k}^{2}} O\left(r^{2}\right) d V_{g} \\
& -\frac{\delta}{2 \lambda_{k} \alpha_{k}} \int_{\partial B_{\delta}\left(x_{k}\right)} e^{\alpha_{k} u_{k}^{2}} d V_{g}+O\left(\frac{\delta}{\beta_{k}^{2}}\right) .
\end{aligned}
$$

Thus the Lemma is proved

Corollary 3.8. We have that

$$
\lim _{k \rightarrow+\infty} \int_{M} e^{\alpha_{k} u_{k}^{2}}=\operatorname{Vol}_{g}(M)+\tau^{2} \lim _{k \rightarrow+\infty} \frac{\lambda_{k}}{\beta_{k}^{2}} .
$$

Moreover we have that

$$
\tau \in(0,1] .
$$

Proof. First of all we have that the sequence $\left(\frac{\lambda_{k}}{\beta_{k}^{2}}\right)_{k}$ is bounded. Indeed using the definition of $\beta_{k}$ we have that

$$
\frac{\lambda_{k}}{\beta_{k}^{2}}=\frac{1}{\lambda_{k}}\left(\int_{M}\left|u_{k}\right| e^{\alpha_{k} u_{k}^{2}} d V_{g}\right)^{2} .
$$

Hence using Jensen's inequality we obtain

$$
\frac{\lambda_{k}}{\beta_{k}^{2}} \leq \frac{1}{\lambda_{k}} \int_{M} e^{\alpha_{k} u_{k}^{2}} d V_{g} \int_{M} u_{k}^{2} e^{\alpha_{k} u_{k}^{2}} d V_{g} .
$$

Thus using the definition of $\lambda_{k}$ we have that

$$
\frac{\lambda_{k}}{\beta_{k}^{2}} \leq \int_{M} e^{\alpha_{k} u_{k}^{2}} d V_{g}
$$

On the other hand one can check easily that

$$
\lim _{k \rightarrow+\infty} \int_{M} e^{\alpha_{k} u_{k}^{2}} d V_{g}=\sup _{u \in \mathcal{H}_{1}} \int_{M} e^{32 \pi^{2} u^{2}} d V_{g}<\infty
$$

Hence we derive that $\left(\frac{\lambda_{k}}{\beta_{k}^{2}}\right)_{k}$ is bounded. So we can suppose without lost of generality that $\left(\frac{\lambda_{k}}{\beta_{k}^{2}}\right)_{k}$ converges.

Now from Lemma 3.7 we have that

$$
\begin{aligned}
& \lim _{k \rightarrow+\infty} \int_{B_{\delta}\left(x_{k}\right)} e^{\alpha_{k} u_{k}^{2}} d V_{g}=16 \pi^{2} \lim _{k \rightarrow+\infty} \frac{\lambda_{k}}{\beta_{k}^{2}}\left(\frac{\delta}{2} \int_{\partial B_{\delta}\left(x_{k}\right)}\left(\beta_{k} U_{k}\right)^{2} d S_{g}\right. \\
& +\delta \int_{\partial B_{\delta}\left(x_{k}\right)} \nabla_{g}\left(\beta_{k} u_{k}\right) \nabla_{g}\left(\beta_{k} U_{k}\right) d S_{g}-2 \int_{\partial B_{\delta}\left(x_{k}\right)}\left(\beta_{k} U_{k}\right) \frac{\partial\left(\beta_{k} u_{k}\right)}{\partial r} \\
& \left.-2 \delta \int_{\partial B_{\delta}\left(x_{k}\right)} \frac{\partial\left(\beta_{k} U_{k}\right)}{\partial r} \frac{\partial\left(\beta_{k} u_{k}\right)}{\partial r} d S_{g}+O(\delta)\right) .
\end{aligned}
$$

So using Lemma 3.6 we obtain

$$
\begin{aligned}
\lim _{k \rightarrow+\infty} \int_{B_{\delta}\left(x_{k}\right)} e^{\alpha_{k} u_{k}^{2}} d V_{g}= & 16 \pi^{2} \lim _{k \rightarrow+\infty} \frac{\lambda_{k}}{\beta_{k}^{2}}\left(\frac{\delta}{2} \int_{\partial B_{\delta}\left(x_{0}\right)}\left|\Delta_{g} G\right|^{2} d S_{g}\right. \\
& +\delta \int_{\partial B_{\delta}(x 0)} \nabla_{g} G \nabla_{g}\left(\Delta_{g} G\right) d S_{g}-2 \int_{\partial B_{\delta}\left(x_{0}\right)} \Delta_{g} G \frac{\partial G}{\partial r} \\
& \left.-2 \delta \int_{\partial B_{\delta}\left(x_{0}\right)} \frac{\partial \Delta_{g} G}{\partial r} \frac{\partial G}{\partial r} d S_{g}+O(\delta)\right) .
\end{aligned}
$$

Moreover by trivial calculations we get

$$
\int_{\partial B_{\delta}\left(x_{0}\right)}\left|\Delta_{g} G\right|^{2} d S_{g}=\frac{\tau^{2}}{8 \pi^{2} \delta}+O(1)
$$




$$
\begin{gathered}
\int_{\partial B_{\delta}\left(x_{0}\right)} \nabla_{g} G \nabla_{g}\left(\Delta_{g} G\right) d S_{g}=-\frac{\tau^{2}}{8 \pi^{2} \delta}+O(1) \\
\int_{\partial B_{\delta}\left(x_{0}\right)} \Delta_{g} G \frac{\partial G}{\partial r}=\frac{\tau^{2}}{16 \pi^{2}}+O(\delta)
\end{gathered}
$$

and

$$
\int_{\partial B_{\delta}\left(x_{0}\right)} \frac{\partial \Delta_{g} G}{\partial r} \frac{\partial G}{\partial r} d S_{g}=-\frac{\tau^{2}}{8 \pi^{2} \delta}+O(1)
$$

Hence with this we obtain

$$
\lim _{k \rightarrow+\infty} \int_{B_{\delta}\left(x_{k}\right)} e^{\alpha_{k} u_{k}^{2}} d V_{g}=\tau^{2} \lim _{k \rightarrow+\infty} \frac{\lambda_{k}}{\beta_{k}^{2}}+O(\delta) .
$$

On the other hand we have that

$$
\int_{M} e^{\alpha_{k} u_{k}^{2}} d V_{g}=\int_{B_{\delta}\left(x_{k}\right)} e^{\alpha_{k} u_{k}^{2}} d V_{g}+\int_{M \backslash B_{\delta}\left(x_{k}\right)} e^{\alpha_{k} u_{k}^{2}} d V_{g}
$$

Moreover by Lemma 3.2 we have that

$$
\int_{M \backslash B_{\delta}\left(x_{k}\right)} e^{\alpha_{k} u_{k}^{2}} d V_{g}=V o l_{g}(M)+o_{k, \delta}(1) .
$$

Thus we derive that

$$
\lim _{k \rightarrow+\infty} \int_{M} e^{\alpha_{k} u_{k}^{2}} d V_{g}=\operatorname{Vol}_{g}(M)+\tau^{2} \lim _{k \rightarrow+\infty} \frac{\lambda_{k}}{\beta_{k}^{2}}+o_{\delta}(1) .
$$

Hence letting $\delta \rightarrow 0$ we obtain

$$
\lim _{k \rightarrow+\infty} \int_{M} e^{\alpha_{k} u_{k}^{2}} d V_{g}=\operatorname{Vol}_{g}(M)+\tau^{2} \lim _{k \rightarrow+\infty} \frac{\lambda_{k}}{\beta_{k}^{2}} .
$$

Now suppose $\tau=0$ then we get

$$
\lim _{k \rightarrow+\infty} \int_{M} e^{\alpha_{k} u_{k}^{2}} d V_{g}=\operatorname{Vol}_{g}(M) .
$$

On the other hand we have that

$$
\lim _{k \rightarrow+\infty} \int_{M} e^{\alpha_{k} u_{k}^{2}} d V_{g}=\sup _{u \in \mathcal{H}_{1}} \int_{M} e^{32 \pi^{2} u^{2}} d V_{g}>\operatorname{Vol}_{g}(M) ;
$$

hence a contradiction. Thus $\tau \neq 0$ and the Corollary is proved.

3.3. Blow-up analysis. In this subsection we perform the Blow-up analysis and show that the asymptotic profile of $u_{k}$ is either the zero function or a standard Bubble.

First of all let us introduce some notations.

We set

$$
r_{k}^{4}=\frac{\lambda_{k}}{\beta_{k} c_{k}} e^{-\alpha_{k} c_{k}^{2}} .
$$

Now for $x \in B^{r_{k}^{-1} \delta}(0)$ with $\delta>0$ small we set

$$
\begin{gathered}
w_{k}(x)=2 \alpha_{k} \beta_{k}\left(u_{k}\left(\exp _{x_{k}}\left(r_{k} x\right)\right)-c_{k}\right) ; \\
v_{k}(x)=\frac{1}{c_{k}} u_{k}\left(\exp _{x_{k}}\left(r_{k} x\right)\right) ; \\
g_{k}(x)=\left(\exp _{x_{k}}^{*} g\right)\left(r_{k} x\right) .
\end{gathered}
$$

Next we define

$$
d_{k}=\frac{c_{k}}{\beta_{k}} \quad d=\lim _{k \rightarrow+\infty} d_{k} .
$$


Proposition 3.9. The following hold:

We have

$$
\text { if } d<+\infty \text { then } w_{k} \rightarrow w(x):=\frac{4}{d} \log \left(\frac{1}{1+\sqrt{\frac{d}{6}}|x|^{2}}\right) \text { in } C_{l o c}^{2}\left(\mathbb{R}^{4}\right) \text {; }
$$

and

$$
\text { if } d=\infty \text { then } \quad w_{k} \rightarrow w=0 \text { in } C_{\text {loc }}^{2}\left(\mathbb{R}^{4}\right) .
$$

Proof. First of all we recall that

$$
g_{k} \rightarrow d x^{2} \text { in } C_{l o c}^{2}\left(\mathbb{R}^{4}\right) .
$$

Since $\left(\frac{\lambda_{k}}{\beta_{k}^{2}}\right),\left(\frac{\beta_{k}}{c_{k}}\right)$ are bounded and $c_{k} \rightarrow+\infty$, then we infer that

$$
r_{k} \rightarrow 0 \text { as } k \rightarrow 0 \text {. }
$$

Now using the Green representation formula for $\Delta_{g}^{2}$ (see Lemma 2.1) we have that

$$
u_{k}(x)=\int_{M} F(x, y) \Delta_{g}^{2} u_{k} d V_{g}(y) \quad \forall x \in M
$$

Now using equation and differentiating with respect to $x$ we obtain that for $m=1,2$

$$
\left|\nabla_{g}^{m} u_{k}(x)\right| \leq \int_{M}\left|\nabla_{g}^{m} F(x, y)\right|\left|\frac{u_{k}}{\lambda_{k}} e^{\alpha_{k} u_{k}^{2}}-\gamma_{k}\right| d V_{g}(y) .
$$

Hence from the fact that $\beta_{k} \gamma_{k}$ is bounded see Lemma 3.4 we get

$$
\left|\nabla_{g}^{m} u_{k}(x)\right| \leq \int_{M}\left|\nabla_{g}^{m} F(x, y)\right|\left|\frac{u_{k}}{\lambda_{k}} e^{\alpha_{k} u_{k}^{2}}\right| d V_{g}(y)+O\left(\beta_{k}^{-1}\right) .
$$

Now for $y_{k} \in B_{L r_{k}}\left(x_{k}\right), L>0$ fixed we write that

$$
\begin{array}{r}
\int_{M}\left|\nabla_{g}^{m} F\left(y_{k}, y\right)\right| \frac{\left|u_{k}\right|}{\lambda_{k}} e^{\alpha_{k} u_{k}^{2}} d V_{g}(y)=O\left(r_{k}^{-m} \int_{M \backslash B_{L r_{k}\left(y_{k}\right)}} \frac{\left|u_{k}\right|}{\lambda_{k}} e^{\alpha_{k} u_{k}^{2}} d V_{g}(y)\right) \\
+O\left(\frac{c_{k}}{\lambda_{k}} e^{\alpha_{k} c_{k}^{2}} \int_{\left.B_{L r_{k}\left(y_{k}\right)} d_{g}\left(y_{k}, y\right)^{-m} d V_{g}(y)\right)}^{=O\left(r_{k}^{-m} \beta_{k}^{-1}\right) .}\right.
\end{array}
$$

thanks to the fact that $\left|u_{k}\right| \leq c_{k}$ to the definition of $r_{k}$.

Now it is not worth remarking that $c_{k}=u_{k}\left(x_{k}\right)$ since we have taken $\tau \geq 0$ (see Lemma 3.4). Hence we have that

$$
w_{k}(x) \leq w_{k}(0)=0 \quad \forall x \in \mathbb{R}^{4} .
$$

So we get from the estimate above that $w_{k}$ is uniformly bounded in $C^{2}(K)$ for every compact subset $K$ of $\mathbb{R}^{4}$. Thus by Arzéla-Ascoli Theorem we infer that

$$
w_{k} \longrightarrow w \in C_{l o c}^{1}\left(\mathbb{R}^{4}\right) .
$$

Clearly $w$ is a Lipschitz function since the constant which bounds the gradient of $w_{k}$ is independent of the compact set $K$.

On the other hand from the Green representation formula we have for $x \in \mathbb{R}^{4}$ fixed and for $L$ big enough such that $x \in B^{L}(0)$

$$
u_{k}\left(\exp _{x_{k}}\left(r_{k} x\right)\right)=\int_{M} F\left(\exp _{x_{k}}\left(r_{k} x\right), y\right) \Delta_{g}^{2} u_{k}(y) d V_{g}(y) .
$$

Now remarking that

$$
u_{k}\left(x_{k}\right)=u_{k}\left(\exp _{x_{k}}\left(r_{k} 0\right)\right)
$$

we have that

$$
u_{k}\left(\exp _{x_{k}}\left(r_{k} x\right)\right)-u_{k}\left(x_{k}\right)=\int_{M}\left(F\left(\exp _{x_{\epsilon}}\left(r_{k} x\right), y\right)-F\left(\exp _{x_{k}}(0), y\right)\right) \Delta_{g}^{2} u_{k}(y) d V_{g}(y) .
$$


Hence using (3.1) we obtain

$$
\begin{aligned}
u_{k}\left(\exp _{x_{k}}\left(r_{k} x\right)\right)-u_{k}\left(x_{k}\right)= & \int_{M}\left(F\left(\exp _{x_{k}}\left(r_{k} x\right), y\right)-F\left(\exp _{x_{k}}(0), y\right)\right) \frac{u_{k}}{\lambda_{k}} e^{\alpha_{k} u_{k}^{2}} d V_{g}(y) \\
& -\int_{M}\left(F\left(\exp _{x_{k}}\left(r_{k} x\right), y\right)-F\left(\exp _{x_{k}}(0), y\right)\right)\left(\gamma_{k}\right) d V_{g}(y) .
\end{aligned}
$$

Now setting

$$
\begin{aligned}
I_{k}(x) & =\int_{B_{L r_{k}}\left(x_{k}\right)}\left(F\left(\exp _{x_{k}}\left(r_{k} x\right), y\right)-F\left(\exp _{x_{k}}(0), y\right)\right) \frac{u_{k}}{\lambda_{k}} e^{\alpha_{k} u_{k}^{2}} d V_{g}(y) ; \\
\mathrm{II}_{k}(x) & =\int_{M \backslash B_{L r_{k}}\left(x_{k}\right)}\left(F\left(\exp _{x_{k}}\left(r_{k} x\right), y\right)-F\left(\exp _{x_{k}}(0), y\right)\right) \frac{u_{k}}{\lambda_{k}} e^{\alpha_{k} u_{k}^{2}} d V_{g}(y)
\end{aligned}
$$

and

we find

$$
\operatorname{III}_{k}(x)=\int_{M}\left(F\left(\exp _{x_{k}}\left(r_{k} x\right), y\right)-F\left(\exp _{x_{k}}(0), y\right)\right)\left(\gamma_{k}\right) d V_{g}(y)
$$

$$
u_{k}\left(\exp _{x_{k}}\left(r_{k} x\right)\right)-u_{k}\left(x_{k}\right)=I_{k}(x)+\mathrm{II}_{k}(x)+\operatorname{III}_{k}(x) .
$$

So using the definition of $w_{k}$ we arrive to

$$
w_{k}=2 \alpha_{k} \beta_{k}\left(I_{k}(x)+\mathrm{II}_{k}(x)+\operatorname{III}_{k}(x)\right) .
$$

Now to continue the proof we consider two cases:

Case 1: $d<+\infty$

First of all let us study each of the terms $2 \alpha_{k} \beta_{k} I_{k}(x), 2 \alpha_{k} \beta_{k} \mathrm{II}_{k}(x), 2 \alpha_{k} \beta_{k} \mathrm{III}_{k}(x)$ separately.

Using the change of variables $y=\exp _{x_{k}}\left(r_{k} z\right)$ we have

$$
\begin{array}{r}
2 \alpha_{k} \beta_{k} I_{k}(x)=\int_{B^{L}(0)}\left(F\left(\exp _{x_{k}}\left(r_{k} x\right), \exp _{x_{k}}\left(r_{k} z\right)\right)-F\left(\exp _{x_{k}}(0), \exp _{x_{k}}\left(r_{k} z\right)\right)\right) \\
\frac{2 \alpha_{k} \beta_{k} u_{k}\left(\exp _{x_{k}}\left(r_{k} z\right)\right)}{\lambda_{k}} e^{\alpha_{k} u_{\epsilon}^{2}\left(\exp _{x_{k}}\left(r_{k} z\right)\right.} r_{k}^{4} d V_{g_{k}}(z) .
\end{array}
$$

Hence using the definition of $r_{k}$ and $v_{k}$ one can check easily that the following holds

$$
\begin{array}{r}
2 \alpha_{k} \beta_{k} I_{k}(x)=2 \alpha_{k} \int_{B^{L}(0)}\left(G\left(\exp _{x_{\epsilon}}\left(r_{k} x\right), \exp _{x_{\epsilon}}\left(r_{k} z\right)\right)-G\left(\exp _{x_{\epsilon}}(0), \exp _{x_{\epsilon}}\left(r_{k} z\right)\right)\right) v_{k}(z) \\
e^{\frac{d_{k}}{2}\left(w_{k}(z)\left(1+v_{k}\right)\right.} d V_{g_{k}}(z) .
\end{array}
$$

Moreover from the asymptotics of the Green function see Lemma 2.1 we have that

$$
2 \alpha_{k} \beta_{k} I_{k}(x)=2 \alpha_{k} \int_{B^{L}(0)}\left(\frac{1}{8 \pi^{2}} \log \frac{|z|}{|x-z|}+K_{k}(x, z)\right) v_{k}(z) e^{\frac{d_{k}}{2}\left(w_{k}(z)\left(1+v_{k}(z)\right)\right)} d V_{g_{k}}(z) .
$$

where

$$
K_{k}(x, z)=\left[K\left(\exp _{x_{k}}\left(r_{k} x\right), \exp _{x_{k}}\left(r_{k} z\right)\right)-\left(K\left(\exp _{x_{k}}(0), \exp _{x_{k}}\left(r_{k} z\right)\right] .\right.\right.
$$

Hence since $K$ is of class $C^{1}$ on $M^{2}$ and $g_{k} \rightarrow d x^{2}$ in $C_{l o c}^{2}\left(\mathbb{R}^{4}\right)$ and $v_{k} \rightarrow 1$ then letting $k \rightarrow+\infty$ we derive

$$
\lim _{k \rightarrow+\infty} 2 \alpha_{k} \beta_{k} I_{k}(x)=8 \int_{B^{L}(0)} \log \frac{|z|}{|x-z|} e^{d w(z)} d z .
$$

Now to estimate $\alpha_{k} \beta_{k} \mathrm{II}_{k}(x)$ we write for $k$ large enough

$$
\begin{aligned}
\alpha_{k} \beta_{k} \mathrm{II}_{k}(x)=\int_{M \backslash B_{L r_{k}}\left(x_{k}\right)} \frac{1}{8 \pi^{2}} & \log \left(\frac{d_{g}\left(\exp _{x_{k}}(0), y\right)}{d_{g}\left(\exp _{x_{k}}\left(r_{k} x\right), y\right)}\right) \frac{2 \alpha_{k} \beta_{k} u_{k}}{\lambda_{k}} e^{\alpha_{k} u_{k}^{2}} d V_{g}(y) \\
& +\int_{M \backslash B_{L r_{k}}\left(x_{k}\right)} \bar{K}_{k}(x, y) \frac{2 \alpha_{k} \beta_{k} u_{k}}{\lambda_{k}} e^{\alpha_{k} u_{k}^{2}} d V_{g}(y),
\end{aligned}
$$

where

$$
\bar{K}_{k}(x, y)=\left(K\left(\exp _{x_{k}}\left(r_{k} x\right), y\right)-K\left(\exp _{x_{k}}(0), y\right)\right) .
$$


Taking the absolute value in both sides of the equality and using the change of variable $y=$ $\exp _{x_{k}}\left(r_{k} z\right)$ and the fact that $K \in C^{1}$ we obtain,

$$
\begin{array}{r}
\left|2 \alpha_{k} \beta_{k} \mathrm{II}_{k}(x)\right| \leq \int_{\mathbb{R}^{4} \backslash B^{L}(0)} 8\left|\log \left(\frac{|z|}{|x-z|}\right)\right| \mid v_{k \mid}(z) e^{\frac{d_{k}}{2}\left(w_{k}(z)\left(1+v_{k}(z)\right)\right)} d V_{g_{k}}(z) \\
\quad+L r_{k} \int_{M \backslash B_{L r_{k}}\left(x_{k}\right)} \frac{2 \alpha_{k} \beta_{k} u_{k}}{\lambda_{k}} e^{\alpha_{k} u_{k}^{2}} d V_{g}(y) .
\end{array}
$$

Hence letting $k \rightarrow+\infty$ we deduce that

$$
\limsup _{k \rightarrow+\infty}\left|2 \alpha_{k} \beta_{k} \mathrm{II}_{k}(x)\right|=o_{L}(1) .
$$

Now using the same method one proves that

$$
2 \alpha_{k} \beta_{k} \operatorname{III}_{k}(x) \rightarrow 0 \text { as } k \rightarrow+\infty .
$$

So we have that

$$
w(x)=\int_{B^{L}(R)} 8 \log \left(\frac{|z|}{|x-z|}\right) e^{d w(z)} d z+\lim _{k \rightarrow+\infty} 2 \alpha_{k} \beta_{k} \mathrm{II}_{k}(x) .
$$

Hence letting $L \rightarrow+\infty$ we obtain that $w$ is a solution of the following integral equation

$$
w(x)=\int_{\mathbb{R}^{4}} 8 \log \left(\frac{|z|}{|x-z|}\right) e^{d w(z)} d z .
$$

Now since $w$ is Lipschitz then the theory of singular integral operator gives that $w \in C^{1}\left(\mathbb{R}^{4}\right)$.

Since

and

$$
\lim _{k \rightarrow+\infty} \int_{B_{L r_{k}}\left(x_{k}\right)} \frac{2 \alpha_{k} \beta_{k} u_{k}}{\lambda_{k}} e^{\alpha_{k} u_{k}^{2}} d V_{g}=64 \pi^{2} \int_{B^{L}(0)} e^{d w(x)} d x
$$

$$
\int_{B_{L r_{k}}\left(x_{k}\right)} \frac{2 \alpha_{k} \beta_{k} u_{k}}{\lambda_{k}} e^{\alpha_{k} u_{k}^{2}} d V_{g} \leq 64 \pi^{2},
$$

then we get

$$
\lim _{L \rightarrow+\infty} \int_{B^{L}(0)} e^{d w(x)} d x=\int_{\mathbb{R}^{4}} e^{d w(x)} d x \leq 1 .
$$

Now setting

$$
\tilde{w}(x)=\frac{d}{4} w(x)+\frac{1}{4} \log \left(\frac{8 \pi^{2} d}{3}\right) ;
$$

we have that $\tilde{w}$ satisfies the following conformally invariant integral equation

$$
\tilde{w}(x)=\int_{\mathbb{R}^{4}} \frac{6}{8 \pi^{2}} \log \left(\frac{|z|}{|x-z|}\right) e^{\tilde{w}(z)} d z+\frac{1}{4} \log \left(\frac{8 \pi^{2} d}{3}\right),
$$

and

$$
\int_{\mathbb{R}^{4}} e^{4 \tilde{w}(x)} d x<+\infty
$$

Hence from the classification result by X.Xu see Theorem 1.2 in [25] we derive that

$$
\tilde{w}(x)=\log \left(\frac{2 \lambda}{\lambda^{2}+\left|x-x_{0}\right|^{2}}\right)
$$

for some $\lambda>0$ and $x_{0} \in \mathbb{R}^{4}$.

¿From the fact that

$$
w(x) \leq w(0)=0 \quad \forall x \in \mathbb{R}^{4}
$$

we obtain

Then we derive

$$
\tilde{w}(x) \leq \tilde{w}(0)=\frac{1}{4} \log \left(\frac{8 \pi^{2} d}{3}\right) \quad \forall x \in \mathbb{R}^{4} .
$$

$$
x_{0}=0, \quad \lambda=2\left(\frac{8 \pi^{2} d}{3}\right)^{-\frac{1}{4}}
$$


Hence by trivial calculations we get

$$
w(x)=\frac{4}{d} \log \left(\frac{1}{1+\sqrt{\frac{d}{6}}|x|^{2}}\right) .
$$

Case 2: $d=+\infty$.

In this case using the same argument we get

$$
\limsup _{k \rightarrow+\infty}\left|\alpha_{k} \beta_{k} \mathrm{II}_{k}(x)\right|=o_{L}(1)
$$

and

Now let us show that

$$
\alpha_{k} \beta_{k} \operatorname{III}_{k}(x)=o_{k}(1)
$$

$$
\alpha_{k} \beta_{k} \mathrm{I}_{k}(x)=o_{k}(1)
$$

By using the same arguments as in Case 1 we get

$$
\alpha_{k} \beta_{k} I_{k}(x)=\int_{B^{L}(0)}\left(\frac{1}{8 \pi^{2}} \log \frac{|z|}{|x-z|}+K_{k}(x, z)\right) v_{k}(z) e^{d_{k}\left(w_{k}(z)\left(1+v_{k}(z)\right)\right)} d V_{g_{k}}(z)
$$

Now since $K$ is $C^{1}$ we need only to show that

$$
\int_{B^{L}(0)} \frac{1}{8 \pi^{2}} \log \frac{|z|}{|x-z|} v_{k}(z) e^{d_{k}\left(w_{k}(z)\left(1+v_{k}(z)\right)\right)} d V_{g_{k}}(z)=o_{k}(1) .
$$

By using the trivial inequality

$$
\int_{B_{L r_{k}\left(x_{k}\right)}} \frac{u_{k}^{2}}{\lambda_{k}} e^{\alpha_{k} u_{k}^{2}} d V_{g} \leq 1
$$

and the change of variables as above, we obtain

$$
\int_{B^{L}(0)} v_{k}^{2}(z) e^{d_{k}\left(w_{k}(z)\left(1+v_{k}(z)\right)\right)} d V_{g_{k}}(z)=O\left(\frac{1}{d_{k}}\right)=o_{k}(1) .
$$

On the other hand using the property of $v_{k}$ one can check easily that

$$
\int_{B^{L}(0)} v_{k}(z) e^{d_{k}\left(w_{k}(z)\left(1+v_{k}(z)\right)\right)} d V_{g_{k}}(z)=\int_{B^{L}(0)} v_{k}^{2}(z) e^{d_{k}\left(w_{k}(z)\left(1+v_{k}(z)\right)\right)} d V_{g_{k}}(z)+o_{k}(1) .
$$

Thus we arrive to

$$
\int_{B^{L}(0)} \frac{1}{8 \pi^{2}} \log \frac{|z|}{|x-z|} v_{k}(z) e^{d_{k}\left(w_{k}(z)\left(1+v_{k}(z)\right)\right)} d V_{g_{k}}(z)=o_{k}(1)
$$

So we get

$$
\alpha_{k} \beta_{k} \mathrm{I}_{k}(x)=o_{k}(1)
$$

Thus letting $k \rightarrow+\infty$, we obtain

$$
w(x)=0 \quad \forall x \in \mathbb{R}^{4} .
$$

Hence the Proposition is proved.

3.4. Capacity estimates. This subsection deals with some capacity-type estimates which allow us to get an upper bound of $\tau^{2} \lim _{k \rightarrow+\infty} \frac{\lambda_{k}}{\beta_{k}^{2}}$. We start by giving a first Lemma to show that we can basically work on Euclidean space in order to get the capacity estimates as already said in the Introduction.

Lemma 3.10. There is a constant $B$ which is independent of $k, L$ and $\delta$ s.t.

$$
\int_{B^{\delta}(0) \backslash B^{L r_{k}(0)}}\left|\left(1-B|x|^{2}\right) \Delta_{0} \tilde{u}_{k}\right|^{2} d x \leq \int_{B_{\delta}\left(x_{k}\right) \backslash B_{L r_{k}}\left(x_{k}\right)}\left|\Delta_{g} u_{k}\right|^{2} d V_{g}+\frac{J_{1}(k, L, \delta)}{\beta_{k}^{2}},
$$

where

Moreover we have that

$$
\tilde{u}(x)=u_{k}\left(\exp _{x_{k}}(x)\right)
$$

$$
\lim _{\delta \rightarrow 0} \lim _{k \rightarrow+\infty} J_{1}(k, L, \delta)=0 .
$$


Proof. First of all by using the definition of $\Delta_{g}$ ie

we get

$$
\Delta_{g}=\frac{1}{\sqrt{|g|}} \partial_{r}\left(\sqrt{|g|} g^{r s} \partial_{s}\right)
$$

$$
\begin{aligned}
\left|\Delta_{g} \beta_{k} u_{k}\right|^{2} & =\left|g^{r s} \beta_{k} \frac{\partial^{2} \tilde{u}_{k}}{\partial x^{r} \partial x^{s}}+O\left(\left|\nabla \beta_{k} \tilde{u}_{k}\right|\right)\right|^{2} \\
& \left.=\left|g^{r s} \beta_{k} \frac{\partial^{2} \tilde{u}_{k}}{\partial x^{r} \partial x^{s}}\right|^{2}+O\left(\left|\nabla^{2} \beta_{k} \tilde{u}_{k}\right|\left|\nabla \beta_{k} \tilde{u}_{k}\right|\right)\right)+O\left(\left(\left|\nabla \beta_{k} \tilde{u}_{k}\right|\right)^{2}\right)
\end{aligned}
$$

On the other hand using the fact that (see Corollary 3.6))

$$
\beta_{k} \tilde{u}_{k} \rightarrow \tilde{G} \text { in } W^{2, p}(M)
$$

where $p \in(1,2)$; and $\tilde{G}(x)=G\left(\exp _{x_{0}}(x)\right)$; we obtain

$$
\begin{gathered}
\int_{B^{\delta}(0) \backslash B^{L r_{k}(0)}} O\left(\left|\nabla^{2} \beta_{k} \tilde{u}_{k} \| \nabla \beta_{k} \tilde{u}_{k}\right|\right)+O\left(\left(\left|\nabla \beta_{k} \tilde{u}_{k}\right|\right)^{2}\right) \\
\leq\left. C|| \tilde{G}\right|_{W^{1,2}\left(B^{\delta}(0) \backslash B^{L r_{k}}(0)\right)} \\
=J_{2}(k, L, \delta)
\end{gathered}
$$

and it is clear that

$$
\lim _{\delta \rightarrow 0} \lim _{k \rightarrow+\infty} J_{2}(k, L, \delta)=0
$$

Now let us estimate $\int_{B^{\delta}(0) \backslash B^{L r_{k}(0)}}\left|g^{r s} \beta_{k} \frac{\partial^{2} \tilde{u}_{k}}{\partial x^{r} \partial x^{s}}\right|^{2}$. To do this, we first write the inverse of the metric in the following form

$$
g^{r s}=\delta^{r s}+A^{r s}
$$

with

We can write

$$
\left|A^{r s}\right| \leq C|x|^{2}
$$

$$
\left|g^{r s} \frac{\partial^{2} \tilde{u}_{k}}{\partial x^{r} \partial x^{s}}\right|^{2}\left|\Delta_{0} \tilde{u}_{k}\right|^{2}+2 \sum_{p, q} A^{p q} \Delta_{0} \tilde{u}_{k} \frac{\partial^{2} \tilde{u}_{k}}{\partial x^{p} \partial x^{q}}+\sum_{r, s, p, q} A^{r s} A^{p q} \frac{\partial^{2} \tilde{u}_{k}}{\partial x^{r} \partial x^{s}} \frac{\partial^{2} \tilde{u}_{k}}{\partial x^{p} \partial x^{q}}
$$

Furthermore we derive

$$
\sum_{p, q} 2 \int_{B^{\delta}(0) \backslash B^{L r_{k}(0)}}\left|A^{p q} \Delta_{0} \tilde{u}_{k} \frac{\partial^{2} \tilde{u}_{k}}{\partial x^{p} \partial x^{q}}\right| d V_{g} \leq C \int_{B^{\delta}(0) \backslash B^{L r_{k}(0)}}\left(|x|^{2}\left|\Delta_{0} \tilde{u}_{k}\right|^{2}+\sum_{p, q}|x|^{2}\left|\frac{\partial^{2} \tilde{u}_{k}}{\partial x^{p} \partial x^{q}}\right|^{2}\right) d x
$$

On the other hand we have that

$$
\begin{array}{r}
\sum_{p, q} \int_{B^{\delta}(0) \backslash B^{L r_{k}(0)}}|x|^{2}\left|\frac{\partial^{2} \tilde{u}_{k}}{\partial x^{p} \partial x^{q}}\right|^{2} d x \int_{B^{\delta}(0) \backslash B^{L r_{k}(0)}}|x|^{2} \frac{\partial^{2} \tilde{u}_{k}}{\partial x^{s} \partial x^{s}} \frac{\partial^{2} \tilde{u}_{k}}{\partial x^{p} \partial x^{p}} d x \\
+\int_{B^{\delta}(0) \backslash B^{L r_{k}(0)}} O\left(\left|\nabla \tilde{u}_{k}\right|\left|\nabla^{2} \tilde{u}_{k}\right|\right) d x \\
+\int_{\partial\left(B^{\delta}(0) \backslash B^{\left.L r_{k}(0)\right)}\right.}|x|^{2} \frac{\partial \tilde{u}_{k}}{\partial x^{q}} \frac{\partial^{2} \tilde{u}_{k}}{\partial x^{p} \partial x^{q}}\left\langle\frac{\partial}{\partial x^{p}}, \frac{\partial}{\partial r}\right\rangle d S \\
+\int_{\partial\left(B^{\delta}(0) \backslash B^{\left.L r_{k}(0)\right)}\right.}|x|^{2} \frac{\partial \tilde{u}_{k}}{\partial x^{q}} \frac{\partial^{2} \tilde{u}_{k}}{\partial x^{p} \partial x^{p}}\left\langle\frac{\partial}{\partial x^{q}}, \frac{\partial}{\partial r}\right\rangle d S .
\end{array}
$$

So setting$$
\frac{J_{3}(k, L, \delta)}{\beta_{k}^{2}}=\int_{B^{\delta}(0) \backslash B^{L r_{k}(0)}} O\left(\left|\nabla \tilde{u}_{k}\right|\left|\nabla^{2} \tilde{u}_{k}\right|\right) d x+\int_{\partial\left(B^{\delta}(0) \backslash B^{\left.L r_{k}(0)\right)}\right.}|x|^{2} \frac{\partial \tilde{u}_{k}}{\partial x^{q}} \frac{\partial^{2} \tilde{u}_{k}}{\partial x^{p} \partial x^{q}}\left\langle\frac{\partial}{\partial x^{p}}, \frac{\partial}{\partial r}\right\rangle d S
$$$$
\left.+\int_{\partial\left(B^{\delta}(0) \backslash B^{\left.L r_{k}(0)\right)}\right.}|x|^{2} \frac{\partial \tilde{u}_{k}}{\partial x^{q}} \frac{\partial^{2} \tilde{u}_{k}}{\partial x^{p} \partial x^{p}}\right)\left\langle\frac{\partial}{\partial x^{q}}, \frac{\partial}{\partial r}\right\rangle d S
$$

We obtain

$$
\sum_{p, q} \int_{B^{\delta}(0) \backslash B^{L r_{k}(0)}}|x|^{2}\left|\frac{\partial^{2} \tilde{u}_{k}}{\partial x^{p} \partial x^{q}}\right|^{2}=\int_{B^{\delta}(0) \backslash B^{L r_{k}(0)}}|x|^{2} \frac{\partial^{2} \tilde{u}_{k}}{\partial x^{q} \partial x^{q}} \frac{\partial^{2} \tilde{u}_{k}}{\partial x^{p} \partial x^{p}} d x+\frac{J_{3}(k, L, \delta)}{\beta_{k}^{2}} .
$$

Moreover we have that

$$
\lim _{\delta \rightarrow 0} \lim _{k \rightarrow+\infty} J_{3}(k, L, \delta)=0
$$


Hence we get

$$
2 \sum_{p, q} \int_{B^{\delta}(0) \backslash B^{L r_{k}(0)}}\left|A^{p q} \frac{\partial^{2} \tilde{u}_{k}}{\partial x^{s} \partial x^{s}} \frac{\partial^{2} \tilde{u}_{k}}{\partial x^{p} \partial x^{q}}\right| \leq C \int_{B^{\delta}(0) \backslash B^{L r_{k}(0)}}|x|^{2}\left|\Delta_{0} \tilde{u}_{k}\right|^{2} d x+\frac{J_{4}(k, L, \delta)}{\beta_{k}^{2}}
$$

with

$$
\lim _{\delta \rightarrow 0} \lim _{k \rightarrow+\infty} J_{4}(k, L, \delta)=0 .
$$

On the other hand using similar arguments we get

$$
\int_{B^{\delta}(0) \backslash B^{L r_{k}(0)}} \sum_{r, s, p, q} A^{r s} A^{p q} \frac{\partial^{2} \tilde{u}_{k}}{\partial x^{r} \partial x^{s}} \frac{\partial^{2} \tilde{u}_{k}}{\partial x^{p} \partial x^{q}} \leq C \int_{B^{\delta}(0) \backslash B^{L r_{k}(0)}}|x|^{4}\left|\Delta_{0} \tilde{u}_{k}\right|^{2} d x+\frac{J_{5}(k, L, \delta)}{\beta_{k}^{2}} .
$$

with

$$
\lim _{\delta \rightarrow 0} \lim _{k \rightarrow+\infty} J_{5}(k, L, \delta)=0
$$

So we arrive to

$$
\int_{B_{\delta}\left(x_{k}\right) \backslash B_{L r_{k}}\left(x_{k}\right)}\left|\Delta_{g} u_{k}\right|^{2} d V_{g} \leq \int_{B^{\delta}(0) \backslash B^{L r_{k}}(0)}\left(1+C|x|^{2}+C|x|^{4}\right)\left|\Delta_{0} \tilde{u}_{k}\right|^{2} d x+\frac{J_{6}(k, L, \delta)}{\beta_{k}^{2}} ;
$$

with

$$
\lim _{\delta \rightarrow 0} \lim _{k \rightarrow+\infty} J_{6}(k, L, \delta)=0
$$

Hence we can find a constant $B_{1}$ independent of $k, L$ and $\delta$ s.t

$$
\int_{B_{\delta}\left(x_{k}\right) \backslash B_{L r_{k}}}\left|\Delta_{g} u_{k}\right|^{2} d V_{g} \geq \int_{B^{\delta}(0) \backslash B^{L r_{k}(0)}}\left(1-B_{1}|x|^{2}\right)\left|\Delta_{0} \tilde{u}_{k}\right|^{2} d x+\frac{J_{7}(k, L, \delta)}{\beta_{k}^{2}} .
$$

So setting

$$
J_{1}(k, L, \delta)=-J_{7}(k, L, \delta) \text { and } B=B_{1}
$$

we have the proved the Lemma.

Next we give a technical Lemma

Lemma 3.11. There exists a sequence of functions $U_{k} \in W^{2,2}\left(B^{\delta}(0) \backslash B^{L r_{k}}(0)\right)$ s.t

$$
\left.U_{k}\right|_{\partial B^{\delta}(0)}=\tau \frac{-\frac{1}{16 \pi^{2}} \log \delta+S_{0}}{\beta_{k}},\left.\quad U_{k}\right|_{\partial B^{L r_{k}(0)}}=\frac{w(L)}{2 \alpha_{k} \beta_{k}}+c_{k} ;
$$

and

Moreover there holds

$$
\left.\frac{\partial U_{k}}{\partial r}\right|_{\partial B_{\delta}(0)}=-\frac{\tau}{8 \pi^{2} \delta \beta_{k}},\left.\quad \frac{\partial U_{k}}{\partial r}\right|_{\partial B^{L r_{k}(0)}}=\frac{w^{\prime}(L)}{2 \alpha_{k} \beta_{k} r_{k}} .
$$

$$
\lim _{\delta \rightarrow 0} \lim _{k \rightarrow+\infty} \beta_{k}^{2}\left(\int_{B^{\delta}(0) \backslash B^{L r_{k}(0)}}\left|\Delta_{0}\left(1-B|x|^{2}\right) U_{k}\right|^{2} d x-\int_{B^{\delta} \backslash B^{L r_{k}(0)}}\left|\left(1-B|x|^{2}\right) \Delta_{0} \tilde{u}_{k}\right|^{2} d x\right)=0 .
$$

Proof. First of all let us set

$$
h_{k}(x)=u_{k}\left(\exp _{x_{k}}\left(r_{k} x\right)\right)
$$

and $u_{k}^{\prime}$ to be the solution of

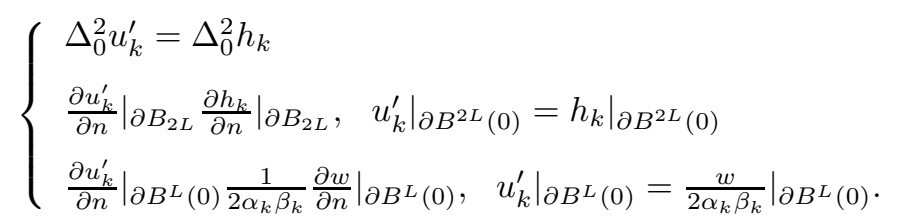

Next let us define

$$
U_{k}^{\prime}= \begin{cases}u_{k}^{\prime}\left(\frac{x}{r_{k}}\right) & L r_{k} \leq|x| \leq 2 L r_{k} \\ \tilde{u}_{k}(x) & 2 L r_{k} \leq|x| .\end{cases}
$$

Clearly we have that

$$
\lim _{k \rightarrow+\infty} \int_{B^{2 L r_{k}(0) \backslash B^{L r_{k}(0)}}}\left(1-B|x|^{2}\right)\left(\left|\Delta_{0} U_{k}^{\prime}\right|^{2}-\left|\Delta_{0} \tilde{u}_{k}\right|^{2}\right) d x=0,
$$


and

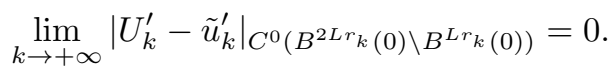

Now let $\eta$ be a smooth function which satisfies

$$
\eta(t)= \begin{cases}1 & t \leq 1 / 2 \\ 0 & t>2 / 3\end{cases}
$$

and set

$$
G_{k}=\eta\left(\frac{|x|}{\delta}\right)\left(\tilde{u}_{k}-\tau S_{0}+\frac{\tau}{8 \pi^{2}} \log |x|\right)-\frac{\tau}{8 \pi^{2}} \log |x|+\tau S_{0} .
$$

Then we have that

$$
G_{k} \rightarrow-\frac{\tau}{8 \pi^{2}} \log |x|+\tau S_{0}+\tau \eta\left(\frac{|x|}{\delta}\right) \tilde{S}_{1}(x)
$$

where $\tilde{S}_{1}(x)=S_{1}\left(\exp _{x_{0}}(x)\right)$.

Furthermore we obtain

$$
\beta_{k} \tilde{u}_{k}-G_{k} \rightarrow \tau\left(1-\eta\left(\frac{|x|}{\delta}\right)\right) S_{1}(x)
$$

then

$$
\left.\lim _{\epsilon \rightarrow 0}\left|\int_{B^{\delta}(0) \backslash B^{\delta / 2}(0)}\right| \Delta_{0} \beta_{k} \tilde{u}_{k}\right|^{2} d x-\int_{B^{\delta}(0) \backslash B^{\delta / 2}(0)}\left|\Delta_{0} G_{k}\right|^{2} d x \mid \leq \Sigma .
$$

where

$$
\begin{aligned}
\Sigma & =\sqrt{\int_{B^{\delta}(0) \backslash B^{\delta / 2}(0)}\left|\Delta_{0}\left(1-\eta\left(\frac{|x|}{\delta}\right)\right) \tilde{S}_{1}(x)\right|^{2} d x \int_{B^{\delta}(0) \backslash B^{\delta / 2}(0)}\left|\Delta_{0}\left(\tilde{G}-\frac{1}{8 \pi^{2}} \log |x|+\eta\left(\frac{|x|}{\delta}\right) \tilde{S}_{1}(x)\right)\right|^{2} d x} \\
& \leq C \delta \sqrt{|\log \delta|}
\end{aligned}
$$

So we get

$$
\left.\lim _{\epsilon \rightarrow 0}\left|\int_{B^{\delta}(0) \backslash B^{\delta / 2}(0)}\right| \Delta_{0} \beta_{k} \tilde{u}_{k}\right|^{2} d x-\int_{B^{\delta}(0) \backslash B^{\delta / 2}(0)}\left|\Delta_{0} G_{k}\right|^{2} d x \mid \leq C \delta \sqrt{|\log \delta|} .
$$

Hence setting

$$
U_{k}= \begin{cases}U_{k}^{\prime}(x) & |x| \leq \frac{\delta}{2} \\ G_{k}(x) & \delta / 2 \leq|x| \leq \delta\end{cases}
$$

we have proved the Lemma.

Proposition 3.12. We have the following holds

$$
\tau^{2} \lim _{k \rightarrow+\infty} \frac{\lambda_{k}}{\beta_{k}^{2}} \leq \frac{\pi^{2}}{6} e^{\frac{5}{3}+32 \pi^{2} S_{0}}
$$

and

$$
d \tau=1
$$

Proof. First using Lemma 3.10 and Lemma 3.11 we get

$$
\int_{B^{\delta}(0) \backslash B^{L r_{k}(0)}}\left|\Delta_{0}\left(1-B|x|^{2}\right) U_{k}\right|^{2} d x \leq 1-\frac{\int_{B_{L}\left(x_{0}\right)}|\Delta w|^{2}+\int_{M \backslash B_{\delta}\left(x_{0}\right)}|\Delta G|^{2}+J_{0}(k, L, \delta)}{\beta_{k}^{2}} .
$$

with

$$
\lim _{\delta \rightarrow 0} \lim _{k \rightarrow+\infty} J_{0}(k, L, \delta)=0 .
$$

Next we will apply capacity to give a lower boundary of $\int_{B^{\delta}(0) \backslash B^{L r_{k}(0)}}\left|\Delta_{0}\left(1-B|x|^{2}\right) U_{k}\right|^{2} d x$.

Hence we need to calculate

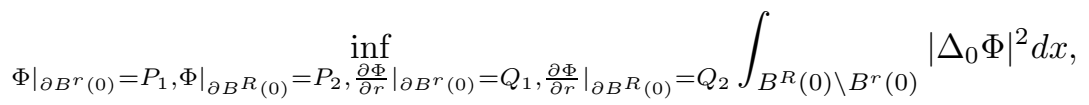


where $P_{1}, P_{2}, Q_{1}, Q_{2}$ are constants.

It is obvious that the infimum is attained by the function $\Phi$ which satisfies

$$
\left\{\begin{array}{l}
\Delta_{0}^{2} \Phi=0 \\
\left.\Phi\right|_{\partial B^{r}(0)}=P_{1},\left.\Phi\right|_{\partial B^{R}(0)}=P_{2},\left.\frac{\partial \Phi}{\partial r}\right|_{\partial B^{r}(0)}=Q_{1},\left.\frac{\partial \Phi}{\partial r}\right|_{\partial B^{R}(0)}=Q_{2} .
\end{array}\right.
$$

Moreover we can require the function $\Phi$ to be of the form

$$
\Phi=A \log r+B r^{2}+\frac{C}{r^{2}}+D,
$$

where $A, B, C, D$ are all constants which satisfies the following linear system of equations

$$
\left\{\begin{array}{l}
A \log r+B r^{2}+\frac{C}{r^{2}}+D=P_{1} \\
A \log R+B R^{2}+\frac{C}{R^{2}}+D=P_{2} \\
\frac{A}{r}+2 B r-2 \frac{C}{r^{3}}=Q_{1} \\
\frac{A}{R}+2 B R-2 \frac{C}{R^{3}}=Q_{2}
\end{array}\right.
$$

Now by straightforward calculations we obtain the explicit expression of $A$ and $B$

$$
\left\{\begin{array}{l}
A=\frac{P_{1}-P_{2}+\frac{\varrho}{2} r Q_{1}+\frac{\varrho}{2} R Q_{2}}{\log r / R+\varrho} \\
B=\frac{-2 P_{1}+2 P_{2}-r Q_{1}\left(1+\frac{2 r^{2}}{R^{2}-r^{2}} \log r / R\right)+R Q_{2}\left(1+\frac{2 R^{2}}{R^{2}-r^{2}} \log r / R\right)}{4\left(R^{2}+r^{2}\right)(\log r / R+\varrho)}
\end{array}\right.
$$

Where $\varrho=\frac{R^{2}-r^{2}}{R^{2}+r^{2}}$. Furthermore we have

$$
\int_{B^{R}(0) \backslash B^{r}(0)}\left|\Delta_{0} \Phi\right|^{2} d x=-8 \pi^{2} A^{2} \log r / R+32 \pi^{2} A B\left(R^{2}-r^{2}\right)+32 \pi^{2} B^{2}\left(R^{4}-r^{4}\right)
$$

In our case in which we have that

$$
\begin{gathered}
R=\delta \quad r=L r_{k}, \\
P_{1}=c_{k}+\frac{w(L)}{2 \alpha_{k} \beta_{k}}+O\left(r_{k} c_{k}\right) \quad P_{2} \frac{-\frac{\tau}{8 \pi^{2}} \log \delta+\tau S_{0}+O(\delta \log \delta)}{\beta_{k}} \\
Q_{1}=\frac{w^{\prime}(L)+O\left(r_{k} c_{k}\right)}{2 \alpha_{k} \beta_{k} r_{k}} \quad Q_{2}=-\frac{\tau+O(\delta \log \delta)}{8 \pi^{2} \beta_{k} \delta} .
\end{gathered}
$$

Then by the formula giving $A$ we obtain by trivial calculations

$$
A=\frac{c_{k}+\frac{N_{k}+\frac{\tau}{8 \pi^{2}} \log \delta}{\beta_{k}}}{-\log \delta+\log L+\frac{\log \frac{\lambda_{k}}{\beta_{k} c_{k}}-\alpha_{k} c_{k}^{2}}{4}+1+O\left(r_{k}^{2}\right)}
$$

where

$$
N_{k}=\frac{w(L)}{2 \alpha_{k}}-\tau S_{0}+\frac{w^{\prime}(L) L}{4 \alpha_{k}}-\frac{\tau}{16 \pi^{2}}+O(\delta \log \delta)+O\left(r_{k} c_{k}^{2}\right) .
$$

Moreover using the the fact that the sequence $\left(\frac{\lambda_{k}}{\beta_{k}^{2}}\right)_{k}$ is bounded it is easily seen that

$$
A=O\left(\frac{1}{c_{k}}\right) .
$$

Furthermore using the formula of $B$ we get still by trivial calculations

$$
B=\frac{-2 c_{k}+\frac{\alpha_{k} c_{k}^{2}}{8 \pi^{2} \beta_{k}} \frac{\tau}{2}+O\left(\frac{1}{\beta_{k}}\right)}{\delta^{2}\left(-\alpha_{k} c_{k}^{2}+\log \frac{\lambda_{k}}{\beta_{k} c_{k}}\right)}
$$

and then

$$
B=O\left(\frac{1}{\beta_{k}}\right) \frac{1}{\delta^{2}}
$$


Now let compute $8 \pi^{2} A^{2} \log r / R$. By using the expression of $A, r$ and $R$, we have that

$$
-8 \pi^{2} A^{2} \log \left(\frac{r}{R}\right)=-8 \pi^{2}\left(\frac{c_{k}+\frac{N_{k}+\frac{\tau}{8 \pi^{2}} \log \delta}{\beta_{k}}}{-\log \delta+\log L+\frac{\log \frac{\lambda_{k}}{\beta_{k} c_{k}}-\alpha_{k} c_{k}^{2}}{4}+1+O\left(r_{k}^{2}\right)}\right)^{2}\left(\frac{\log \frac{\lambda_{k}}{\beta_{k} c_{k}}-\alpha_{k} c_{k}^{2}}{4}-\log \delta+\log L\right)
$$

Now using the relation

$$
\begin{array}{r}
\left(\frac{\alpha_{k} c_{k}^{2}}{4}\right)^{2}\left(1-\frac{1}{\alpha_{k} c_{k}^{2}}\left(-4 \log \delta+4 \log L+\log \frac{\lambda_{k}}{\beta_{k} c_{k}}+4+O\left(r_{k}^{2}\right)\right)\right)^{2}= \\
\left(-\log \delta+\log L+\frac{\log \frac{\lambda_{k}}{\beta_{k} c_{k}}-\alpha_{k} c_{k}^{2}}{4}+1+O\left(r_{k}^{2}\right)\right)^{2}
\end{array}
$$

we derive

$$
\begin{aligned}
-8 \pi^{2} A^{2} \log \left(\frac{r}{R}\right)=-8 \pi^{2}\left(\frac{c_{k}+\frac{N_{k}+\frac{\tau}{8 \pi^{2}} \log \delta}{\beta_{k}}}{\frac{\alpha_{k} c_{k}^{2}}{4}}\right)^{2}\left(1-\frac{1}{\alpha_{k} c_{k}^{2}}(-4 \log \delta+\right. & \left.\left.4 \log L+\log \frac{\lambda_{k}}{\beta_{k} c_{k}}+4+O\left(r_{k}^{2}\right)\right)\right)^{-2} \\
& \times\left(\frac{\log \frac{\lambda_{k}}{\beta_{k} c_{k}}-\alpha_{k} c_{k}^{2}}{4}-\log \delta+\log L\right) .
\end{aligned}
$$

On the other hand using Taylor expansion we have the following identity

$$
\begin{array}{r}
\left(1-\frac{1}{\alpha_{k} c_{k}^{2}}\left(-4 \log \delta+4 \log L+\log \frac{\lambda_{k}}{\beta_{k} c_{k}}+4+O\left(r_{k}^{2}\right)\right)\right)^{-2}=1+2 \frac{\log \frac{\lambda_{k}}{\beta_{k} c_{k}}+4-4 \log \delta+4 \log L}{\alpha_{k} c_{k}^{2}} \\
+O\left(\frac{\log ^{2} c_{k}}{c_{k}^{4}}\right) ;
\end{array}
$$

hence we get

$$
\begin{array}{r}
-8 \pi^{2} A^{2} \log \left(\frac{r}{R}\right)=-8 \pi^{2}\left(\frac{c_{k}+\frac{N_{k}+\frac{\tau}{8 \pi^{2}} \log \delta}{\beta_{k}}}{\frac{\alpha_{k} c_{k}^{2}}{4}}\right)^{2}\left(\frac{\log \frac{\lambda_{k}}{\beta_{k} c_{k}}-\alpha_{k} c_{k}^{2}}{4}-\log \delta+\log L\right) \\
\times\left(1+2 \frac{\log \frac{\lambda_{k}}{\beta_{k} c_{k}}+4-4 \log \delta+4 \log L}{\alpha_{k} c_{k}^{2}}+O\left(\frac{\log ^{2} c_{k}}{c_{k}^{4}}\right)\right)
\end{array}
$$

On the other hand using the relation

$$
\begin{gathered}
-8 \pi^{2}\left(\frac{c_{k}+\frac{N_{k}+\frac{\tau}{8 \pi^{2}} \log \delta}{\beta_{k}}}{\frac{\alpha_{k} c_{k}^{2}}{4}}\right)^{2}\left(\frac{\log \frac{\lambda_{k}}{\beta_{k} c_{k}}-\alpha_{k} c_{k}^{2}}{4}-\log \delta+\log L\right)= \\
\frac{32 \pi^{2}}{\alpha_{k}} \frac{1}{c_{k}^{2}}\left(c_{k}+\frac{N_{k}+\frac{\tau}{8 \pi^{2}} \log \delta}{\beta_{k}}\right)^{2}\left(1-\frac{\log \frac{\lambda_{k}}{\beta_{k} c_{k}}-4 \log \delta+4 \log L}{\alpha_{k} c_{k}^{2}}\right)
\end{gathered}
$$

we obtain

$$
\begin{array}{r}
-8 \pi^{2} A^{2} \log \left(\frac{r}{R}\right)=\frac{32 \pi^{2}}{\alpha_{k}} \frac{1}{c_{k}^{2}}\left(c_{k}+\frac{N_{k}+\frac{\tau}{8 \pi^{2}} \log \delta}{\beta_{k}}\right)^{2}\left(1+2 \frac{\log \frac{\lambda_{k}}{\beta_{k} c_{k}}+4-4 \log \delta+4 \log L}{\alpha_{k} c_{k}^{2}}+O\left(\frac{\log ^{2} c_{k}}{c_{k}^{4}}\right)\right) \\
\times\left(1-\frac{\log \frac{\lambda_{k}}{\beta_{k} c_{k}}-4 \log \delta+4 \log L}{\alpha_{k} c_{k}^{2}}\right)
\end{array}
$$

Moreover using again the trivial relation

$$
\begin{array}{r}
\left(1+2 \frac{\log \frac{\lambda_{k}}{\beta_{k} c_{k}}+4-4 \log \delta+4 \log L}{\alpha_{k} c_{k}^{2}}+O\left(\frac{\log ^{2} c_{k}}{c_{k}^{4}}\right)\right)\left(1-\frac{\log \frac{\lambda_{k}}{\beta_{k} c_{k}}-4 \log \delta+4 \log L}{\alpha_{k} c_{k}^{2}}\right)= \\
\left(1+\frac{\log \frac{\lambda_{k}}{\beta_{k} c_{k}}+8-4 \log \delta+4 \log L}{\alpha_{k} c_{k}^{2}}+O\left(\frac{\log ^{2} c_{k}}{c_{k}^{4}}\right)\right)
\end{array}
$$


we arrive to

$$
-8 \pi^{2} A^{2} \log \left(\frac{r}{R}\right)=\frac{32 \pi^{2}}{\alpha_{k}} \frac{1}{c_{k}^{2}}\left(c_{k}+\frac{N_{k}+\frac{\tau}{8 \pi^{2}} \log \delta}{\beta_{k}}\right)^{2}\left(1+\frac{\log \frac{\lambda_{k}}{\beta_{k} c_{k}}+8-4 \log \delta+4 \log L}{\alpha_{k} c_{k}^{2}}+O\left(\frac{\log ^{2} c_{k}}{c_{k}^{4}}\right)\right)
$$

On the other hand one can check easily that the following holds

$$
\begin{array}{r}
\left(c_{k}+\frac{N_{k}+\frac{\tau}{8 \pi^{2}} \log \delta}{\beta_{k}}\right)^{2}\left(1+\frac{\log \frac{\lambda_{k}}{\beta_{k} c_{k}}+8-4 \log \delta+4 \log L}{\alpha_{k} c_{k}^{2}}+O\left(\frac{\log ^{2} c_{k}}{c_{k}^{4}}\right)\right)= \\
\left(c_{k}^{2}+\frac{\log \frac{\lambda_{k}}{\beta_{k} c_{k}}+8-4 \log \delta+4 \log L}{\alpha_{k}}+2 c_{k} \frac{N_{k}+\frac{\tau}{8 \pi^{2}} \log \delta}{\beta_{k}}+O\left(\frac{\log c_{k}}{c_{k}^{2}}\right)+O\left(\frac{1}{\beta_{k}^{2}}\right)\right)
\end{array}
$$

thus we obtain

$$
\begin{array}{r}
-8 \pi^{2} A^{2} \log \left(\frac{r}{R}\right)=\frac{32 \pi^{2}}{\alpha_{k}} \frac{1}{c_{k}^{2}}\left(c_{k}^{2}+\frac{\log \frac{\lambda_{k}}{\beta_{k} c_{k}}+8-4 \log \delta+4 \log L}{\alpha_{k}}+2 c_{k} \frac{N_{k}+\frac{\tau}{8 \pi^{2}} \log \delta}{\beta_{k}}\right) \\
+\frac{32 \pi^{2}}{\alpha_{k}} \frac{1}{c_{k}^{2}}\left(O\left(\frac{\log c_{k}}{c_{k}^{2}}\right)+O\left(\frac{1}{\beta_{k}^{2}}\right)\right)
\end{array}
$$

Furthermore using the relation

$$
\begin{gathered}
\left(c_{k}^{2}+\frac{\log \frac{\lambda_{k}}{\beta_{k} c_{k}}+8-4 \log \delta+4 \log L}{\alpha_{k}}+2 c_{k} \frac{N_{k}+\frac{\tau}{8 \pi^{2}} \log \delta}{\beta_{k}}+O\left(\frac{\log c_{k}}{c_{k}^{2}}\right)+O\left(\frac{1}{\beta_{k}^{2}}\right)\right)= \\
\left(c_{k}^{2}+\frac{1}{\alpha_{k}} \log \frac{\lambda_{k}}{\beta_{k} c_{k}}-\frac{4}{\alpha_{k}} \log \delta+\frac{1}{4 \pi^{2}} d_{k} \tau \log \delta+2 d_{k} N_{k}+\frac{4 \log L}{\alpha_{k}}+\frac{8}{\alpha_{k}}+o_{k}(1)\right)
\end{gathered}
$$

we get

$$
\begin{array}{r}
-8 \pi^{2} A^{2} \log \left(\frac{r}{R}\right)=\frac{32 \pi^{2}}{\alpha_{k}^{2}} \frac{1}{c_{k}^{2}}\left(c_{k}^{2}+\frac{1}{\alpha_{k}} \log \frac{\lambda_{k}}{\beta_{k} c_{k}}-\frac{4}{\alpha_{k}} \log \delta+\frac{1}{4 \pi^{2}} d_{k} \tau \log \delta+2 d_{k} N_{k}+\frac{4 \log L}{\alpha_{k}}+\frac{8}{\alpha_{k}}\right) \\
+\frac{32 \pi^{2}}{\alpha_{k}^{2}} \frac{1}{c_{k}^{2}} o_{k}(1)
\end{array}
$$

Next we will evaluate $\int_{M \backslash B_{\delta}\left(x_{0}\right)} \Delta_{g} G \Delta_{g} G d V_{g}$. We have that by Green formula

$$
\int_{M \backslash B_{\delta}\left(x_{0}\right)} \Delta_{g} G \Delta_{g} G d V_{g}=\int_{M \backslash B_{\delta}\left(x_{0}\right)} G \Delta_{g}^{2} G d V_{g}-\int_{\partial B_{\delta}\left(x_{0}\right)} \frac{\partial G}{\partial r} \Delta_{g} G+\int_{\partial B_{\delta}\left(x_{0}\right)} G \frac{\partial \Delta_{g} G}{\partial r} .
$$

Thus using the equation solved by $G$ we get

$$
\begin{array}{r}
\int_{M \backslash B_{\delta}\left(x_{0}\right)} \Delta_{g} G \Delta_{g} G d V_{g}=-\frac{\tau}{\mu(M)} \int_{M \backslash B_{\delta}(p)} G d V_{g}-\frac{\tau^{2}}{64 \pi^{4}} \int_{\partial B_{\delta}\left(x_{0}\right)} \frac{\partial(-\log r)}{\partial r} \Delta_{0}(-\log r) \\
+\int_{\partial B_{\delta}\left(x_{0}\right)}\left(-\frac{\tau}{8 \pi^{2}} \log r+S_{0}\right) \frac{\partial \Delta_{0}\left(-\frac{\tau}{8 \pi^{2}} \log r\right)}{\partial r}+O(\delta \log \delta)
\end{array}
$$

Hence we obtain

$$
\int_{M \backslash B_{\delta}\left(x_{0}\right)} \Delta_{g} G \Delta_{g} G d V_{g}=-\frac{\tau^{2}}{16 \pi^{2}}-\frac{\tau^{2}}{8 \pi^{2}} \log \delta+\tau^{2} S_{0}+O(\delta \log \delta),
$$

Now let us set

$$
P(L)=\int_{B^{L}(0)}\left|\Delta_{0} w\right|^{2} d x /\left(2 \times 32 \pi^{2}\right)^{2} .
$$

Hence using (3.12), (3.13), (3.14), we derive that

$$
\begin{array}{r}
\frac{32 \pi^{2}}{\alpha_{k}}\left(c_{k}^{2}+\frac{1}{\alpha_{k}} \log \frac{\lambda_{k}}{\beta_{k} c_{k}}-\frac{4}{\alpha_{k}} \log \delta+\frac{1}{4 \pi^{2}} d_{k} \tau \log \delta+2 d_{k} N_{k}+\frac{4 \log L}{\alpha_{k}}+\frac{8}{\alpha_{k}}\right) \\
\leq c_{k}^{2}\left(1-\frac{P(L)-\frac{\tau^{2}}{16 \pi^{2}}-\frac{\tau^{2}}{8 \pi^{2}} \log \delta+\tau S_{0}+O(\delta \log \delta)+o_{k, \delta}(1)}{\beta_{k}^{2}}\right)+\delta^{2} O\left(c_{k}^{2} A B\right)+\delta^{4} O\left(c_{k}^{2} B^{2}\right) .
\end{array}
$$


Moreover by isolating the term $\frac{32 \pi^{2}}{\alpha_{k}^{2}} \log \frac{\lambda_{k}}{\beta_{k} c_{k}}$ in the left and transposing all the other in the right we get

$$
\begin{array}{r}
\frac{32 \pi^{2}}{\alpha_{k}^{2}} \log \frac{\lambda_{k}}{\beta_{k} c_{k}} \leq \frac{1}{8 \pi^{2}}\left(d_{k}^{2} \tau^{2}-\frac{64}{\alpha_{k}} d_{k} \tau+\left(\frac{32 \pi}{\alpha_{k}}\right)^{2}\right) \log \delta-\frac{32 \pi^{2}}{\alpha_{k}}\left(2 d_{k} N_{k}+\frac{4 \log L}{\alpha_{k}}+\frac{8}{\alpha_{k}}\right) \\
-d_{k}^{2}\left(P(L)+\tau S_{0}-\frac{\tau^{2}}{16 \pi^{2}}+O(\delta \log \delta)+o_{k}(1)\right)+\delta^{2} O\left(c_{k}^{2} A B\right)+\delta^{4} O\left(c_{k}^{2} B^{2}\right) .
\end{array}
$$

Hence using the trivial identity

$$
\log \frac{\lambda_{k}}{\beta_{k}^{2}}=\log \frac{\lambda_{k}}{\beta_{k} c_{k}}+\log d_{k}
$$

we get

$$
\begin{aligned}
\frac{32 \pi^{2}}{\alpha_{k}^{2}} \log \frac{\lambda_{k}}{\beta_{k}^{2}} \leq & \frac{1}{8 \pi^{2}}\left(d_{k}^{2} \tau^{2}-\frac{64}{\alpha_{k}} d_{k} \tau+\left(\frac{32 \pi}{\alpha_{k}}\right)^{2}\right) \log \delta-\frac{32 \pi^{2}}{\alpha_{k}}\left(2 d_{k} N_{k}+\frac{2+4 \log L}{\alpha_{k}}+\frac{2}{\alpha_{k}}\right) \\
& -d_{k}^{2}\left(P(L)+\tau S_{0}-\frac{\tau^{2}}{16 \pi^{2}}+O(\delta \log \delta)+o_{k}(1)\right)+\frac{32 \pi^{2}}{\alpha_{k}^{2}} \log d_{k}+O\left(d_{k}^{2}\right) .
\end{aligned}
$$

Now suppose $d=+\infty$, letting $\delta \rightarrow 0$, then we have that

$$
\lim _{k \rightarrow+\infty} \log \frac{\lambda_{k}}{\beta_{k}^{2}}=-\infty
$$

thus we derive

$$
\lim _{k \rightarrow+\infty} \frac{\lambda_{k}}{\beta_{k}^{2}}=0
$$

Hence using Corollary 3.8 we obtain a contradiction. So $d$ must be finite.

On the other hand one can check easily that the following holds

$$
\frac{32 \pi^{2}}{\alpha_{k}^{2}} \log \frac{\lambda_{k}}{\beta_{k}^{2}} \leq \frac{1}{8 \pi^{2}}\left(d_{k} \tau-\frac{32 \pi^{2}}{\alpha_{k}}\right)^{2} \log \delta+O(1)\left(d_{k}^{2}+d_{k}+\log d_{k}\right)+O(1) .
$$

Hence we derive

$$
d_{k} \tau \rightarrow 1
$$

otherwise we reach the same contradiction. So we have that

$$
d \tau=1
$$

Hence by using this we can rewrite $B$ as follows

$$
B=\frac{-2 c_{k}+\delta\left(-\frac{1}{8 \pi^{2} c_{k} \delta} 2 \frac{-\alpha_{k} c_{k}^{2}}{4}\right)+O\left(1 / c_{k}\right)}{\delta^{2}\left(-\alpha_{k} c_{k}^{2}\right)+O(1)}=\frac{o_{k}(1)}{c_{k}} .
$$

Thus we obtain

$$
32 \pi^{2} A B\left(R^{2}-r^{2}\right)+32 \pi^{2} B^{2}\left(R^{4}-r^{4}\right)=\frac{o_{k}(1)}{c_{k}^{2}} .
$$

On the other hand since $d<+\infty$, we have that by Lemma 3.9

$$
w=-\frac{4 \log \left(1+\sqrt{\frac{d}{6}} \pi|x|^{2}\right)}{d} .
$$

Moreover by trivial calculations we get

$$
P(L)=\frac{1}{96 d^{2} \pi^{2}}+\frac{\log \left(1+\sqrt{\frac{d}{6}} \pi L^{2}\right)}{16 d^{2} \pi^{2}} .
$$

Furthermore by taking the limit as $k \rightarrow+\infty$ in (3.15) we obtain

$$
\lim _{k \rightarrow+\infty} \log \frac{\lambda_{k}}{\beta_{k} c_{k}} \leq-\frac{25}{3}+4 d \tau+2 d^{2} \tau^{2}+32 \pi^{2} S_{0}+\frac{4 \sqrt{\frac{d}{6}} \pi L^{2}}{1+\sqrt{\frac{d}{6}} \pi L^{2}}+2 \log \left(1+\sqrt{\frac{d}{6}} \pi L^{2}\right)-4 \log L
$$


Now letting $L \rightarrow+\infty$, we get

$$
\lim _{k \rightarrow+\infty} \log \frac{\lambda_{k}}{\beta_{k} c_{k}} \leq \frac{5}{3}-\log 6+\log \pi^{2}+\log d .
$$

Hence by remarking the trivial identity

$$
\lim _{k \rightarrow+\infty} \frac{\lambda_{k}}{\beta_{k} c_{k}} \frac{1}{d} \lim _{k \rightarrow+\infty} \frac{\lambda_{k}}{\beta_{k}^{2}}
$$

we get

$$
\tau^{2} \lim _{k \rightarrow+\infty} \frac{\lambda_{k}}{\beta_{k}^{2}} \leq \frac{\pi^{2}}{6} e^{\frac{5}{3}+32 \pi^{2} S_{0}} .
$$

So the proof of the proposition is done.

3.5. The test function. This Subsection deals with the construction of some test functions in order to reach a contradiction.

Now let $\epsilon>0, c>0, L>0$ and set

$$
f_{\epsilon}(x)= \begin{cases}c+\frac{\Lambda+B d_{g}\left(x, x_{0}\right)^{2}-4 \log \left(1+\lambda\left(\frac{d_{g}\left(x, x_{0}\right)}{\epsilon}\right)^{2}\right)}{64 \pi^{2} c}+\frac{S(x)}{c} & d_{g}\left(x, x_{0}\right) \leq L \epsilon \\ \frac{G(x)}{c} & d_{g}\left(x, x_{0}\right)>L \epsilon\end{cases}
$$

where

$$
\lambda=\frac{\pi}{\sqrt{6}}, \quad B=-\frac{4}{L^{2} \epsilon^{2}\left(1+\lambda L^{2}\right)}
$$

and

$$
\Lambda=-64 \pi^{2} c^{2}-B L^{2} \epsilon^{2}-8 \log (L \epsilon)+4 \log \left(1+\lambda L^{2}\right) .
$$

Proposition 3.13. We have that for $\epsilon$ small, there exist suitable $c$ and $L$ such that

$$
\int_{M}\left|\Delta_{g} f_{\epsilon}\right|^{2} d V_{g}=1
$$

and

$$
\limsup _{\epsilon \rightarrow 0} \int_{M} e^{32 \pi^{2}\left(f_{\epsilon}-\bar{f}_{\epsilon}\right)^{2}} d V_{g}>\operatorname{Vol}(M)+\frac{\pi^{2}}{6} e^{\frac{5}{3}+32 \pi^{2} S_{0}} .
$$

Proof. First of all using the expansion of $g$ in normal coordinates we get

$$
\int_{B_{L \epsilon}\left(x_{0}\right)}\left|\Delta_{g} f_{\epsilon}\right|^{2} d V_{g} \int_{B^{L \epsilon}(0)}\left|\Delta_{0} \tilde{f}_{\epsilon}\right|^{2}\left(1+O(L \epsilon)^{2}\right) d x+\int_{B^{L \epsilon}(0)} O\left(r^{2}\left|\nabla_{0} \tilde{f}_{\epsilon}\right|^{2}\right) d x
$$

where

$$
\tilde{f}_{\epsilon}(x)=f_{\epsilon}\left(\exp _{x_{0}}(x)\right) .
$$

On the other hand by direct calculations owe obtain

$$
\int_{B^{L \epsilon}(0)}\left|\Delta_{0} \tilde{f}_{\epsilon}\right|^{2} d x=\frac{12+\lambda L^{2}\left(30+\lambda L^{2}\left(21+\lambda L^{2}\right)\right)+6\left(1+\lambda L^{3}\right)^{3} \log \left(1+\lambda L^{2}\right)}{96 c^{2}\left(1+\lambda L^{2}\right)^{3} \pi^{2}}
$$

Hence we arrive to

$$
\begin{aligned}
\int_{B_{L \epsilon}\left(x_{0}\right)}\left|\Delta_{g} f_{\epsilon}\right|^{2} d V_{g} & =\left(1+O(L \epsilon)^{2}\right) \frac{12+\lambda L^{2}\left(30+\lambda L^{2}\left(21+\lambda L^{2}\right)\right)+6\left(1+\lambda L^{3}\right)^{3} \log \left(1+\lambda L^{2}\right)}{96 c^{2}\left(1+\lambda L^{2}\right)^{3} \pi^{2}} \\
& =\frac{\frac{1}{3}+4 \log \left(1+\lambda L^{2}\right)+O\left(\frac{1}{L^{2}}\right)+O\left((L \epsilon)^{2} \log L \epsilon\right)}{32 c^{2} \pi^{2}}
\end{aligned}
$$

Furthermore, by direct computation, we have

$$
\int_{B^{L \epsilon}(0)} r^{2}\left|\nabla_{0} \tilde{f}_{\epsilon}\right|^{2} d x=O\left(\frac{L^{4} \epsilon^{4}}{c^{2}}\right) .
$$

Moreover using Green formula we get

$$
\begin{aligned}
\int_{M \backslash B_{L \epsilon}\left(x_{0}\right)}\left|\Delta_{g} G\right|^{2} d V_{g} & =\int_{M \backslash B_{L \epsilon}\left(x_{0}\right)} G d V_{g}-\int_{\partial B_{L \epsilon}\left(x_{0}\right)} \frac{\partial G}{\partial r} \Delta_{g} G d S_{g}+\int_{\partial B_{L \epsilon}} G \frac{\partial \Delta_{g} G}{\partial r} d S_{g} \\
& =-\frac{1}{16 \pi^{2}}+S_{0}-\frac{\log L \epsilon}{8 \pi^{2}}+O(L \epsilon \log L \epsilon)
\end{aligned}
$$


Now let us find a condition to have $\int_{M}\left|\Delta_{g} f_{\epsilon}\right|^{2} d V_{g}=1$. By trivial calculations we can see that it is equivalent to

$$
\frac{1}{32 \pi^{2} c^{2}}\left(-\frac{5}{3}+2 \log \left(1+\lambda L^{2}\right)+32 \pi^{2} S_{0}-4 \log L \epsilon+O\left(\frac{1}{L^{2}}\right)+O(L \epsilon \log L \epsilon)\right)=1 .
$$

i.e.

$$
32 \pi^{2} c^{2}=-\frac{5}{3}+2 \log \left(1+\lambda L^{2}\right)+32 \pi^{2} S_{0}-4 \log L \epsilon+O\left(\frac{1}{L^{2}}\right)+O(L \epsilon \log L \epsilon) .
$$

Hence by (3.16) $\Lambda$ take the following form

$$
\Lambda=\frac{10}{3}-64 \pi^{2} S_{0}+O\left(\frac{1}{L^{2}}\right)+O(L \epsilon \log L \epsilon) .
$$

On the other hand it is easily seen that

$$
\int_{B_{L \epsilon}\left(x_{0}\right)} f_{\epsilon} d V_{g}=O\left(c(L \epsilon)^{4}\right)
$$

and

hence

$$
\int_{M \backslash B_{L \epsilon}\left(x_{0}\right)} f_{\epsilon} d V_{g}=-\int_{B_{L \epsilon}} \frac{G}{c}=O\left(\frac{(L \epsilon)^{4} \log L \epsilon}{c}\right) .
$$

$$
\bar{f}_{\epsilon}=O\left(c(L \epsilon)^{4}\right) .
$$

Furthermore by trivial calculations one gets that in $B_{L \epsilon}\left(x_{0}\right)$

$$
\begin{aligned}
\left(f_{\epsilon}-\bar{f}_{\epsilon}\right)^{2} & \geq c^{2}+\frac{2}{64 \pi^{2}}\left(\Lambda+B r^{2}-4 \log \left(1+\lambda\left(\frac{r}{\epsilon}\right)^{2}\right)+64 \pi^{2} S_{0}+O(L \epsilon)+O\left(c^{2}(L \epsilon)^{4}\right)\right) \\
& =c^{2}+\frac{5}{48 \pi^{2}}-\frac{\log \left(1+\lambda(r / \epsilon)^{2}\right)}{8 \pi^{2}}+O\left(\frac{1}{L^{2}}\right)+O(L \epsilon \log L \epsilon)+O\left(c^{2}(L \epsilon)^{4}\right)
\end{aligned}
$$

hence

$$
\begin{aligned}
\int_{B_{L \epsilon}\left(x_{0}\right)} e^{32 \pi^{2}\left(f_{\epsilon}-\bar{f}_{\epsilon}\right)^{2}} d V_{g} & \geq\left(1+O(L \epsilon)^{2}\right) \int_{B_{L \epsilon}\left(x_{0}\right)} e^{32 \pi^{2}\left(c^{2}+\frac{5}{48 \pi^{2}}-\frac{\log \left(1+\lambda(r / \epsilon)^{2}\right.}{8 \pi^{2}}\right)+O\left(\frac{1}{L^{2}}\right)+O(L \epsilon \log L \epsilon)+O\left(c^{2}(L \epsilon)^{4}\right)} d x \\
& =\epsilon^{4} e^{\frac{10}{3}+32 \pi^{2} c^{2}+O\left(\frac{1}{L^{2}}\right)+O(L \epsilon \log L \epsilon)+O\left(c^{2}(L \epsilon)^{4}\right)}\left(\pi^{2} \frac{L^{6}}{1+\lambda L^{6}}+O(L \epsilon)^{2}\right) \\
& =\epsilon^{4} e^{\frac{10}{3}+32 \pi^{2} c^{2}} \pi^{2}\left(1+O\left(\frac{1}{L^{2}}\right)+O(L \epsilon \log L \epsilon)+O(L \epsilon)^{2}\right) \\
& =\frac{\pi^{2}}{6} e^{\frac{5}{3}+32 \pi^{2} S_{0}}\left(1+O(L \epsilon \log L \epsilon)+O\left(\frac{1}{L^{2}}\right)+O\left(c^{2}(L \epsilon)^{4}\right)\right) .
\end{aligned}
$$

on the other hand

$$
\begin{aligned}
\int_{M \backslash B_{L \epsilon}\left(x_{0}\right)} e^{32 \pi^{2}\left(f_{\epsilon}-\bar{f}_{\epsilon}\right)^{2}} d V_{g} & \geq \int_{M \backslash B_{L \epsilon}\left(x_{0}\right)}\left(1+32 \pi^{2}\left(f_{\epsilon}-\bar{f}_{\epsilon}\right)^{2}\right) d V_{g} \\
& \geq \operatorname{Vol}\left(M \backslash B_{L \epsilon}\left(x_{0}\right)\right)+\frac{\int_{M \backslash B_{L \epsilon}\left(x_{0}\right)} 32 \pi^{2} G^{2} d V_{g}+O\left(c(L \epsilon)^{4}\right)}{c^{2}} \\
& =\operatorname{Vol}(M)+\frac{\int_{M} 32 \pi^{2} G^{2} d V_{g}}{c^{2}}+O(L \epsilon)^{4} \log L \epsilon
\end{aligned}
$$

Thus we arrive to

$$
\begin{aligned}
\int_{M} e^{32 \pi^{2}\left(f_{\epsilon}-\bar{f}_{\epsilon}\right)^{2}} d V_{g} \geq \operatorname{Vol}(M)+ & \frac{\pi^{2}}{6} e^{\frac{5}{3}+32 \pi^{2} S_{0}}+\frac{\int_{M \backslash B_{L \epsilon}\left(x_{0}\right)} 32 \pi^{2} G^{2} d V_{g}}{c^{2}} \\
& +O(L \epsilon \log (L \epsilon))+O\left(\frac{1}{L^{2}}\right)+O\left(c^{2}(L \epsilon)^{4}\right)
\end{aligned}
$$

and factorizing by $\frac{1}{c^{2}}$ we get

$$
\begin{aligned}
\int_{M} e^{32 \pi^{2}\left(f_{\epsilon}-\bar{f}_{\epsilon}\right)^{2}} d V_{g} \geq & \operatorname{Vol}(M)+\frac{\pi^{2}}{6} e^{\frac{5}{3}+32 \pi^{2} S_{0}} \\
& +\frac{1}{c^{2}}\left(\int_{M} 32 \pi^{2} G^{2} d V_{g}+O\left(c^{2} L \epsilon \log (L \epsilon)\right)+O\left(\frac{c^{2}}{L^{2}}\right)+O\left(c^{4}(L \epsilon)^{4}\right)\right) .
\end{aligned}
$$


On the other hand setting

$$
L=\log \frac{1}{\epsilon}
$$

we get

$$
O\left(c^{2} L \epsilon \log (L \epsilon)\right)+O\left(\frac{c^{2}}{L^{2}}\right)+O\left(c^{4}(L \epsilon)^{4}\right) \rightarrow 0 \text { as } \epsilon \rightarrow 0 .
$$

Hence the Proposition is proved.

3.6. Proof of Theorem 1.1. This small subsection is concerned about the proof of Theorem 1.1. First of all by corollary we have that

$$
\lim _{k \rightarrow+\infty} \int_{M} e^{\alpha_{k} u_{k}^{2}}=\operatorname{Vol}_{g}(M)+\tau^{2} \lim _{k \rightarrow+\infty} \frac{\lambda_{k}}{\beta_{k}^{2}}
$$

with $\tau \neq 0$.

On the other hand from Proposition 3.12 we get

$$
\tau^{2} \lim _{k \rightarrow+\infty} \frac{\lambda_{k}}{\beta_{k}^{2}} \leq \frac{\pi^{2}}{6} e^{\frac{5}{3}+32 \pi^{2} S_{0}} .
$$

Hence we obtain

$$
\lim _{k \rightarrow+\infty} \int_{M} e^{\alpha_{k} u_{k}^{2}} \leq \operatorname{Vol}_{g}(M)+\frac{\pi^{2}}{6} e^{\frac{5}{3}+32 \pi^{2} S_{0}} .
$$

Thus using the relation

$$
\lim _{k \rightarrow+\infty} \int_{M} e^{\alpha_{k} u_{k}^{2}} d V_{g}=\sup _{u \in \mathcal{H}_{1}} \int_{M} e^{32 \pi^{2} u^{2}} d V_{g}
$$

we derive

$$
\sup _{u \in \mathcal{H}_{1}} \int_{M} e^{32 \pi^{2} u^{2}} d V_{g} \leq \operatorname{Vol}_{g}(M)+\frac{\pi^{2}}{6} e^{\frac{5}{3}+32 \pi^{2} S_{0}} .
$$

On the other hand from Proposition 3.13 we have the existence of a family of function $f_{\epsilon}$ such that

$$
\int_{M}\left|\Delta_{g} f_{\epsilon}\right|^{2} d V_{g}=1
$$

and

$$
\limsup _{\epsilon \rightarrow 0} \int_{M} e^{32 \pi^{2}\left(f_{\epsilon}-\bar{f}_{\epsilon}\right)^{2}} d V_{g}>\operatorname{Vol}(M)+\frac{1}{6} e^{\frac{5}{3}+32 \pi^{2} S_{0}} \pi^{2} .
$$

Hence we reach a contradiction. So the proof of Theorem 1.1 is completed.

\section{Proof of Theorem 1.2}

As already said in the Introduction, in this brief Section we will explain how the proof of Theorem 1.1 remains valid for Theorem 1.2.

First of all we remark that all the analysis above have been possible due to the following facts 1) $\int_{M}\left|\Delta_{g} u\right|^{2} d V_{g}$ is an equivalent norm to the standard norm of $H^{2}(M)$ on $\mathcal{H}_{1}$. 2)

The existence of the Green function for $\Delta_{g}^{2}$.

3)

The result of Fontana.

On the other hand we have a counterpart of 2) and 3). Moreover it is easy to see that $\left\langle P_{g}^{4} u, u\right\rangle$ is also an equivalent norm to the standard norm of $H^{2}(M)$ on $\mathcal{H}_{2}$. Notice that for a blowing-up sequence $u_{k}$ we have that

$$
\left\langle P_{g}^{4} u_{k}, u_{k}\right\rangle=\int_{M}\left|\Delta_{g} u_{k}\right|^{2} d V_{g}+o_{k}(1) ;
$$

then it is easy to see that the same proof is valid up to the subsection of test functions. Notice that (4.1) holds for the test functions $f_{\epsilon}$, then it is easy to see that continuing the same proof we get Theorem 1.2. 


\section{REFERENCES}

[1] Adams D.R., A sharp inequality of J.Moser for higher order derivatives, Ann. math 128 (1988) 385-398.

[2] Adimurthi and O.Druet, Blow up analysis in dimension 2 and a sharp form of Trudinger Moser inequality, Comm. PDE 29 No 1-2, (2004), 295-322.

[3] Beckner W., Sharp Sobolev inequalities on the sphere and the Moser-trudinger inequality, Ann. Math 138(1) (1993) 213-242.

[4] Chang S.Y.A., The Moser-Trudinger inequality and applications to some problems in conformal geometry,in Nonlinear Partial differential equations in Differential Geometry (Park City, UT, 1992) IAS/Park City Mathematics series, Vol. 2(American Mathematical society, Providence, rI, 1996), pp. 65-125.

[5] Carleson L, Chang S.Y.A., On the existence of an extremal function for an inequality of J.Moser, Bull. Sci. Math 110 (1986) 113-127.

[6] Chang S.Y.A., Yang P.C.,Extremal metrics of zeta functional determinants on 4-manifolds, ann. of Math. 142(1995), 171-212.

[7] Chang S.Y.A., Gursky M.J., Yang P.C.,A conformally invariant sphere theorem in four dimensions, Publ. Math. Inst. Hautes etudes Sci. 9892003), 105-143.

[8] Djadli Z., Malchiodi A., Existence of conformal metrics with constant Q-curvature, to appear in ann. of Math.

[9] Fontana L., Sharp bordeline sobolev inequalities on compact Riemmanian manifolds, comment. Math.Helv. 68 (1993) 415-454.

[10] Flucher M., Extremal functions for Trudinger-Moser type inequality in 2 dimensions, Comment. math.Helv. 67 (1992) 471-497.

[11] Gursky M., The principal eigenvalue of a conformally invariant differential operator, with an application to semilinear elliptic PDE, Comm. Math. Phys. 207-1 (1999), 131-143.

[12] Li J., Li Y., Liu P., The Q-curvature on a 4-dimensional Riemannian manifold $(M, g)$ with $\int_{M} Q d V_{g}=8 \pi^{2}$, preprint.

[13] Li Y., Liu P., Moser-Trudinger inequality on the boundary of compact of compact Riemannian surface, Math. Z. 250 (2) (2005) 363-386.

[14] Li Y., Moser-Trudinger inequality on compact Riemannian manifolds of dimension two, J.Partial Differential equations 14(2) (2001) 1289-1319.

[15] Li Y., The extremal functions for Moser-trudinger inequality on compact Riemannian manifolds, Sci. China Series A. Math. 48 (2005) 18-648.

[16] Lin K.C.,Extremal functions for moser's inequality, trans. Amer. Math. Soc. 348 (1996) 2663-2671.

[17] Malchiodi A., Compactness of solutions to some geometric fourth-order equations, J. Reine Angew. Math., to appear.

[18] Malchiodi A., Ndiaye C.B., Some existence results for the Toda system on closed surfaces, preprint, 2005

[19] Moser J., A Sharp form of an inequality of Trudinger, Ind. univ. math. j. 20 (1971) 1077-1091.

[20] Ndiaye C.B., Constant Q-curvature metrics in arbitrary dimension preprint 2006.

[21] Paneitz S., A quartic conformally covariant differential operator for arbitrary pseudo-Riemannian manifolds, preprint, 1983.

[22] Pohozaev S.I.,: The Sobolev embedding in the case $p l=n$. Proceedings of the Technical Scientific Conference on Advances of Scientific Research 1964-1965, Mathematics Section, 158-170, Moskov. Energet. Inst., Moscow, 1965

[23] Struwe M.,: Critical points of embedding of $H_{0}^{1, n}$ into Orlicz space, Ann. Inst. Henri., 5(5):425-464, 1988. 
[24] Trudinger N.S.,on embedding into orlicz space and some applications, J. Math. mech. 17 (1967) 473-484.

[25] Xu Xingwang., Uniqueness and non-existence theorems for conformally invariant equations, Journal of Functional Analysis. 222(2005) 1-28.

(Yuxiang Li) ICTP, Mathematics Section, Strada Costiera 11, 34014 Trieste, Italy

E-mail address, yuxiang li: liy@ictp.it

(Cheikh Birahim Ndiaye) S.I.S.S.A., Via Beirut 2-4, 34014, Trieste, Italy

E-mail address, Cheikh Birahim Ndiaye: ndiaye@sissa.it 